San Jose State University

SJSU ScholarWorks

Master's Theses

Master's Theses and Graduate Research

Fall 2015

\title{
Effects of Acute Exercise, Mindfulness Meditation, and Mindfulness Meditation Neurofeedback on Stroop Performance
}

Claire Evelyn Balgemann

San Jose State University

Follow this and additional works at: https://scholarworks.sjsu.edu/etd_theses

\section{Recommended Citation}

Balgemann, Claire Evelyn, "Effects of Acute Exercise, Mindfulness Meditation, and Mindfulness Meditation Neurofeedback on Stroop Performance" (2015). Master's Theses. 4621.

DOI: https://doi.org/10.31979/etd.j63m-fmu3

https://scholarworks.sjsu.edu/etd_theses/4621

This Thesis is brought to you for free and open access by the Master's Theses and Graduate Research at SJSU ScholarWorks. It has been accepted for inclusion in Master's Theses by an authorized administrator of SJSU ScholarWorks. For more information, please contact scholarworks@sjsu.edu. 


\author{
A Thesis \\ Presented to \\ The Faculty of the Graduate Program in Human Factors and Ergonomics
}

San José State University

In Partial Fulfillment

of the Requirements for the Degree

Master of Science

by

Claire E. Balgemann

December 2015 
(C) 2015

Claire E. Balgemann

ALL RIGHTS RESERVED 
The Designated Thesis Committee Approves the Thesis Titled

EFFECTS OF ACUTE EXERCISE, MINDFULNESS MEDITATION, AND MINDFULNESS MEDITATION NEUROFEEDBACK ON STROOP PERFORMANCE

by

Claire E. Balgemann

APPROVED FOR THE GRADUATE PROGRAM IN HUMAN FACTORS \& ERGONOMICS

DEPARTMENT OF INDUSTRIAL AND SYSTEMS ENGINEERING

SAN JOSÉ STATE UNIVERSITY

December 2015
Dr. Emily H. Wughalter
Department of Kinesiology
Dr. Sean Laraway
Department of Psychology
Dr. David Schuster
Department of Psychology 


\begin{abstract}
EFFECTS OF ACUTE EXERCISE, MINDFULNESS MEDITATION, AND MINDFULNESS MEDITATION NEUROFEEDBACK ON STROOP PERFORMANCE by Claire E. Balgemann
\end{abstract}

Empirical research has demonstrated that acute aerobic physical exercise, neurofeedback, and mindfulness meditation independently have positive effects on executive performance. Technology has enabled a new intervention that combines neurofeedback and mindfulness meditation; however its effects on executive function are unknown. The aim in the present study was to understand how executive function may be enhanced in healthy individuals. Forty-two participants were randomly assigned to one of three intervention groups: acute physical exercise, mindfulness meditation, or mindfulness meditation neurofeedback delivered via the Muse brain-sensing headband. Participants completed 50 trials of the Stroop task, a well-established measure of executive function. A statistically significant one-way ANOVA revealed that participants made fewer errors after undergoing the mindfulness meditation neurofeedback intervention than they did following acute physical exercise. These findings demonstrate that mindfulness meditation neurofeedback may boost aspects of executive function that relate to lower Stroop task error rates. 


\section{ACKNOWLEDGMENTS}

I would first like to thank my thesis advisor, Dr. Emily H. Wughalter, for providing intellectual guidance while allowing me the freedom to develop my project independently. Thank you for your insightful comments throughout the versions of this thesis and for lending direction and cohesion to the final product. To my committee members, Dr. Sean Laraway and Dr. David Schuster, my sincere appreciation for your encouragement, ideas, and support. This work is substantially stronger as a result of your input and guidance.

Thank you to Dr. Yasser Dessouky for helping me to find a space to complete a full day's worth of data collection that happened to be scheduled during the university's campus orientation day. Your willingness to assist an unknown graduate student saved the day.

I would also like to thank my parents, who have always selflessly backed my education and whatever unusual pursuits attracted my interest. Thank you for giving me the freedom and confidence to explore so many different paths.

Finally, my profound thanks to Güneş Kayacik, without whom I can honestly say I never would have gone back to school at all, let alone completed this thesis. Thank you for your encouragement, optimism, and patience throughout the planning, writing, analyzing, and agonizing that came with this thesis. 
For Scott 


\section{Table of Contents}

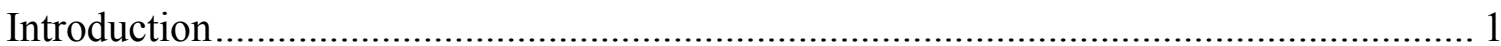

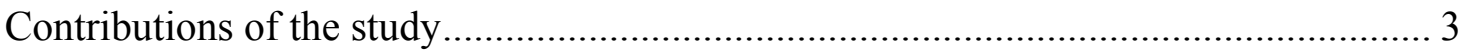

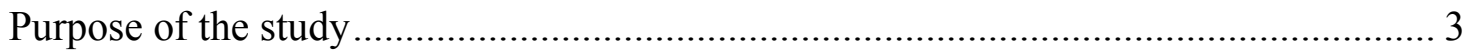

Definition of Terms.......................................................................................... 4

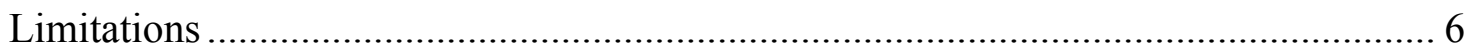

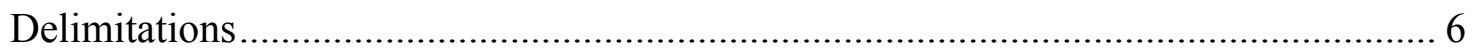

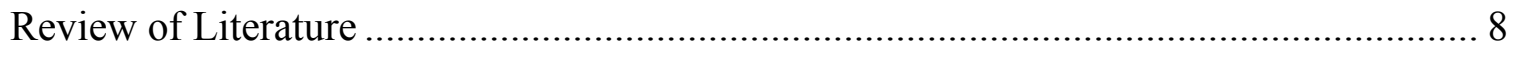

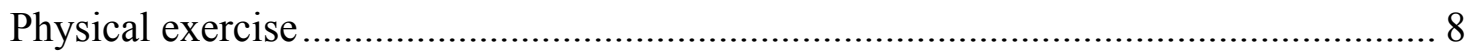

Exercise type as a moderating variable..................................10

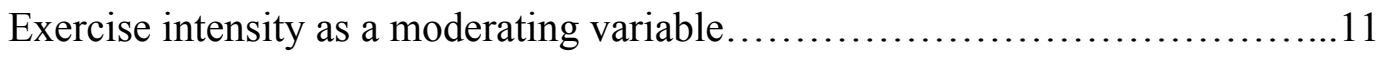

Participant fitness level as a moderating variable............................13

Exercise activity as a moderating variable................................14

Timing of cognitive task as a moderating variable......................... 15

Type of cognitive task as a moderating variable............................ 16

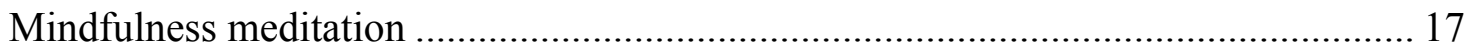

Historical background................................................ 18

Practical applications of mindfulness meditation........................... 19

Mindfulness meditation and cognitive performance........................21

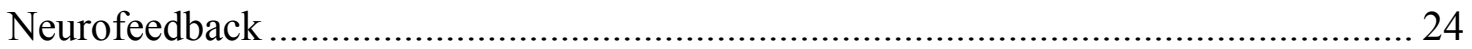

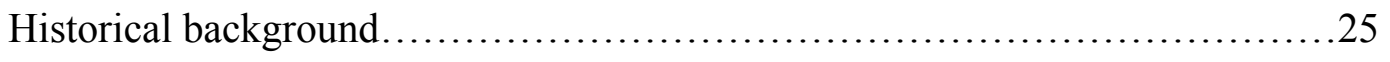

Neurofeedback procedures.............................................26

Neurofeedback and cognitive performance..............................28

Mindfulness meditation neurofeedback .................................................................. 29 


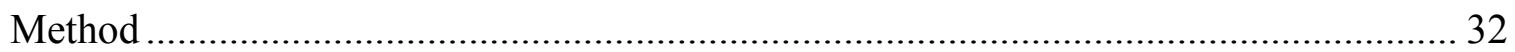

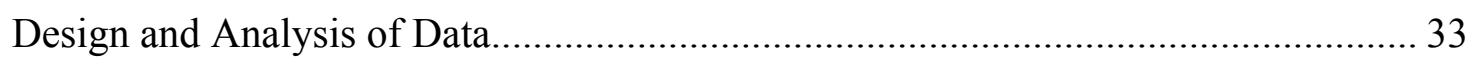

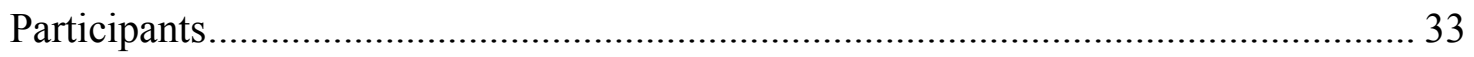

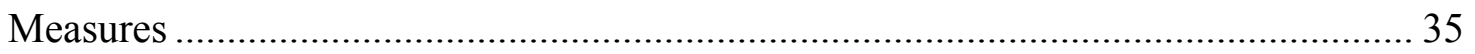

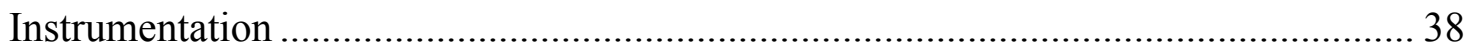

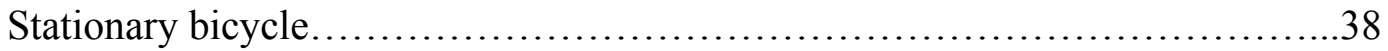

Heart rate monitor................................................... 39

Muse brain-sensing headband............................................ 39

Muse Calm application............................................40

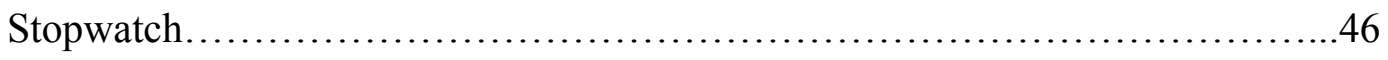

Tally counter...................................................46

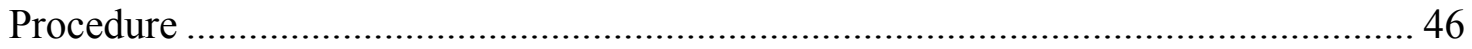

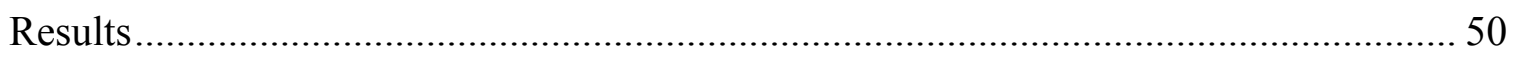

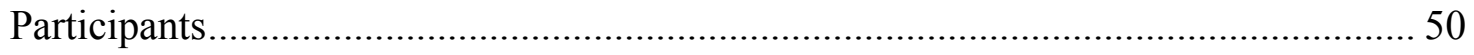

Effect of study interventions on Stroop task time................................................ 54

Effect of study interventions on Stroop task number of errors .............................. 55

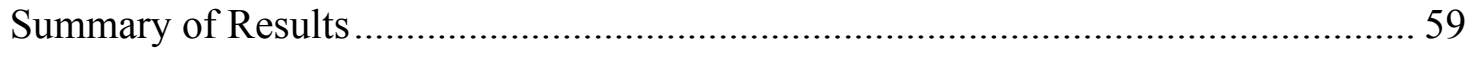

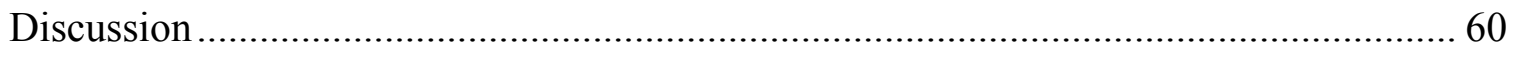

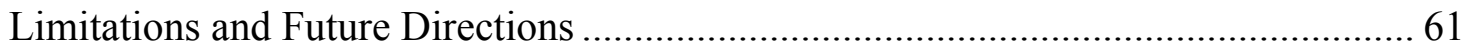

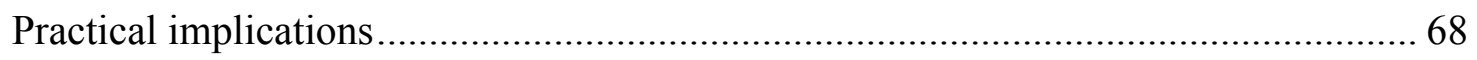

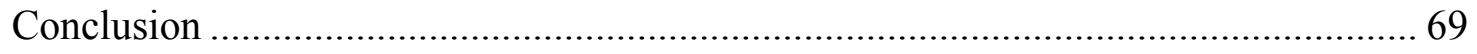




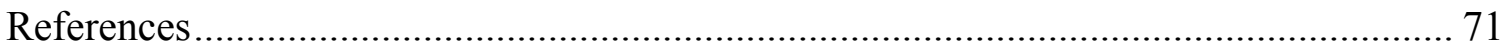

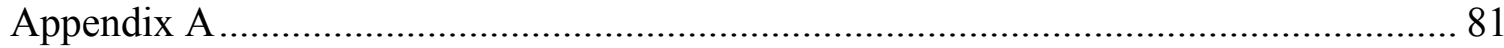

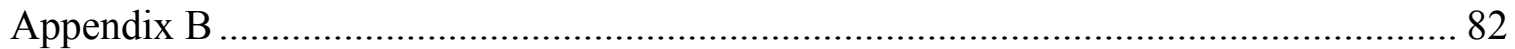

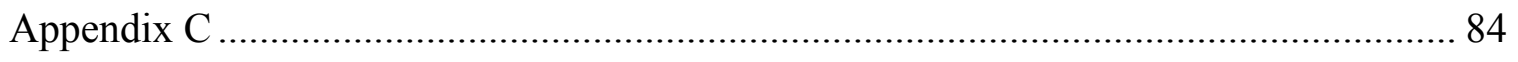

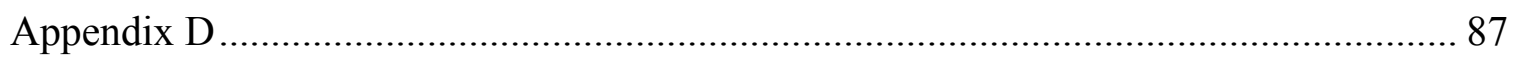




\section{List of Figures}

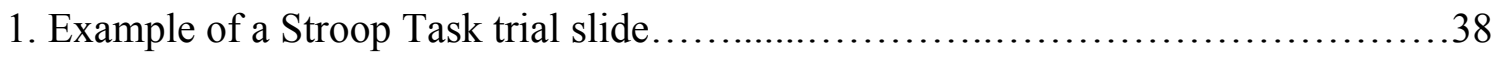

2. Image of the stationary bicycle that was used in the acute physical exercise group, the

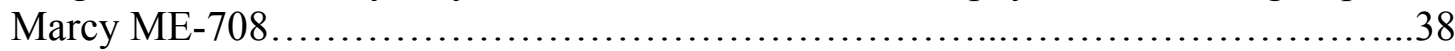

3. Image of the heart rate monitor that was used in the acute physical exercise group, the MIO 0021UX-BLK2 Drive+ Strapless Heart Rate Monitor Watch....................39

4. Image of the Muse Brain-Sensing Headband device..................................40

5. Screenshot of the Muse Calm application Session Options screen.....................41

6. Screenshot of the Muse Calm application calibration screen.........................42

7. Screenshot of the Muse Calm application thought calibration instruction screen........43

8. Screenshot of the Muse Calm application thought calibration countdown screen.....43

9. Screenshot of the Muse Calm application mindfulness mediation instruction screen...44

10. Screenshot of the Muse Calm application mindfulness meditation countdown

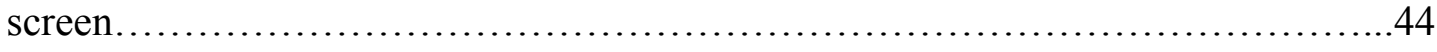

11. Screenshot of the Muse Calm application mindfulness meditation countdown screen with cloud and waves, indicating active brainwaves.............................45

12. Flow chart showing study procedures for the three intervention groups...............47

13. Frequency distribution of Stroop task number of errors by intervention group.......59

14. Example of post-session graphical output from the Muse device ....................68

15. Example of post-session percentage output from the Muse device..................69 


\section{List of Tables}

1. Participant Demographics by Intervention Group.............................52

2. Participant Self-reported Frequency and Duration of Moderate Intensity Exercise by Intervention Group......................................................... 54

3. Participant Self-Reported Frequency and Duration of High Intensity Exercise by

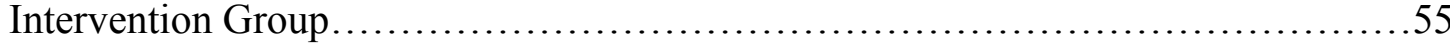

4. Descriptive Statistics for Stroop Task Time by Intervention Group.................56

5. Descriptive Statistics for Stroop Task Number of Errors by Intervention Group......57

6. Pairwise Comparisons for Stroop Task Number of Errors.......................59

7. Chi-Square Contingency Table Expected and Actual Stroop Number of Errors.......63 


\section{Chapter 1}

\section{Introduction}

Executive function is a high-level cognitive ability that encompasses several foundational cognitive functions including inhibition, task switching, attention, and working memory (Etnier \& Chang, 2009). In a world with an ever-increasing number of attentional demands, executive function is an essential skill. It helps an individual to manage multiple informational inputs simultaneously, filter distractions, and pivot between strategies. Executive function is an important part of cognitive performance that has been studied prolifically in both healthy and clinical populations (Alvarez \& Emory, 2006).

Three methods for improving executive function that are established are: mindfulness meditation (Chan \& Woollacott, 2007; Deepeshwar, Vinchurkar, Visweswaraiah, \& Nagendra, 2015; Moore \& Malinowski, 2009; Wenk-Sormaz, 2005), neurofeedback (Bink, van Nieuwenhuizen, Popma, Bongers, \&van Boxtel, 2014), and acute physical exercise (Barella, Etnier, \& Chang, 2010; Chang et al., 2014; Labelle, Bosquet, Mekary, \& Bherer, 2013; Sibley et al., 2006). Mindfulness meditation is a mental practice where an individual maintains a calm, non-judgmental mind while focusing on breath or other sensations in the body (Wallace \& Shapiro, 2006). Neurofeedback is an operant conditioning procedure in which an electroencephalograph (EEG) or similar device reads a user's brainwaves and then communicates data to users in visual or audial cues (Vernon, 2005). 
A new intervention has been invented that combines mindfulness meditation and neurofeedback. Mindfulness meditation neurofeedback is delivered by way of a lightweight, consumer-grade EEG headband that senses five brainwave frequencies and produces real-time neurofeedback on a user's mobile device. The mindfulness meditation neurofeedback device is called the Muse brain-sensing headband, and is produced by InteraXon Inc. The device is advertised to consumers as a way to improve focus, productivity, composure, and overall happiness with its use (Muse by InteraXon Inc., 2015). Through neurofeedback, Muse teaches users mindfulness meditation processes, including focusing attention on the breath and bringing attention back to the breath when neurofeedback indicates mind wandering (Muse by InteraXon Inc., 2015). The present study investigated the effects of mindfulness meditation neurofeedback on executive performance. Investigation of this intervention is novel, and thus one goal of this pilot study was to learn how best to test mindfulness meditation neurofeedback as an intervention.

This study investigated the effects of three interventions on executive performance, mindfulness meditation neurofeedback, mindfulness meditation, and acute physical exercise, as measured by the Stroop task (Stroop, 1935). Etnier and Chang (2009) cited the Stroop task as one of the three most frequently used tests of executive function. The Stroop task was selected for this study because of its sensitivity to the physiological effects of acute aerobic exercise as well as attentional training, which is similar to mindfulness meditation and neurofeedback (Chang et al., 2012; Lambourne \& Tomporowski, 2010; Markowska, 2013; Moore \& Malinowski, 2009). 


\section{Contributions of the study}

Few studies have investigated both mindfulness meditation and neurofeedback. Lai, MacNeil, and Frewen (2014) and Chow (2014) compared the individual effects of mindfulness meditation and neurofeedback in healthy populations, but their research did not include a combined mindfulness meditation neurofeedback condition. Therefore, further research is needed to understand the effects of mindfulness meditation neurofeedback, which may be one contribution of this work.

In addition to investigating the effects of mindfulness meditation neurofeedback, this study examined how acute aerobic exercise and mindfulness meditation affect Stroop performance. While several studies have independently studied Stroop affects following acute physical exercise (Barella, Etnier, \& Chang, 2010; Chang et al., 2014; Labelle, Bosquet, Mekary, and Bherer, 2013; Sibley, Etnier, \& Masurier, 2006) or mindfulness meditation (Chan \& Woollacott, 2007; Deepeshwar, Vinchurkar, Visweswaraiah, \& Nagendra, 2015; Moore \& Malinowski, 2009; Wenk-Sormaz, 2005), none have specifically looked at their effects on Stroop task time and number of errors.

\section{Purpose of the study}

The purpose of this study was to determine:

1. How does intervention (acute physical exercise, mindfulness meditation, or mindfulness meditation neurofeedback) affect Stroop task time?

2. How does intervention (acute physical exercise, mindfulness meditation, or mindfulness meditation neurofeedback) affect Stroop task number of errors? 


\section{Definition of Terms}

Accuracy. In the present study, accuracy was measured in the number of errors a participant made during 50 trials of the Stroop task. The fewer errors made, the more accurate the participant.

Acute physical exercise. Acute physical exercise refers to a single bout of exercise, as compared to chronic exercise which is exercise repeated over a period of time. Acute physical exercise was used as one of three interventions. A 20-minute bout of moderate-intensity exercise was completed on a stationary bicycle.

Executive function. Executive function is a high-level cognitive ability that controls basic cognitive functions such as inhibition, working memory, attention, and task switching. In the present study, cognitive performance was measured more specifically as executive performance via the Stroop task, one of the three most frequently used tests of executive function (Etnier \& Chang, 2009).

Mindfulness meditation. Mindfulness meditation is the practice of being aware of the present moment without placing judgment on internal thoughts or feelings. Mindfulness meditation was one of three intervention groups used in this study. For the purpose of this study, mindfulness meditation practices were introduced to participants via the Muse device. Both the mindfulness meditation and the mindfulness meditation neurofeedback groups received mindfulness meditation instructions via the Muse device. Feedback from the Muse device was then turned off for the mindfulness meditation group, but left on for the mindfulness meditation neurofeedback group. 
Neurofeedback. Neurofeedback is an operant conditioning training method in which participants receive audiovisual feedback in accordance with changes in their brainwave patterns with the ultimate goal of learning to control the brainwaves. For this purpose of this study, neurofeedback was combined with mindfulness meditation in a new intervention created by the Muse device. Mindfulness meditation neurofeedback was one of three intervention groups used in this study. The mindfulness meditation neurofeedback group received mindfulness meditation training via the Muse device, similar to the mindfulness meditation group, but then also received audiovisual feedback from the Muse device when the brainwaves became more active or calmer.

Response time. Response time is the total amount of time it takes for an individual to react to a stimulus. For the purpose of this study, response time will refer to the time it takes for participants' to verbalize and answer to a Stroop task trial.

Stroop task. The Stroop task, also known as the Stroop color-word test, is task in which the name of a color is printed in a different color (e.g., the word "red" printed in the color blue). Participants must say the color that the word is, not what the word reads. The Stroop task is an established measure of cognitive performance, specifically executive function. This study measured the amount of time a participant took to complete the Stroop task and the amount of errors made. For the purpose of this study, the Stroop task consisted of 50 trials with four words and four colors (red, green, blue, or yellow). 


\section{Limitations}

The following limitations were recognized:

1. Participants were recruited from introductory psychology classes and advertisements placed around a large university in the Western United States. Students who attend a large university in the Western United States may exhibit traits that the general population does not, limiting the external validity of this study.

2. Resource limitations resulted in a small sample size of 42 total participants, 14 per three interventions. The small sample size may limit the generalizability of the results of this study to the population as a whole and reduce statistical power.

3. While research suggests that a single bout of intervention may produce measurable effects (Kluetsch et al., 2014; Ros et al., 2013), limiting the three interventions in this study to a single 20-minute session may have resulted in effects that are different than what would be found in a longitudinal study.

\section{Delimitations}

The following delimitations were placed upon the study:

1. Participants in this study were all between the ages of 18 and 69 years so that they qualified for the Physical Activity Readiness Questionnaire (PAR-Q; See Appendix A).

2. Participants had to be healthy enough to exercise as determined by answering negatively to all questions on the PAR-Q. 
3. Participants were required to meet or exceed the American College of Sports Medicine's (ACSM, 2014) aerobic exercise frequency and intensity recommendations to be selected to participate in this study. The ACSM recommends 150 minutes or more moderate intensity exercise per week or 75 minutes or more of high intensity exercise per week (ACSM, 2014). This delimitation was in place to ensure that participants were accustomed to regular exercise and fit enough to participate in exercise without injury.

4. Individuals who practiced mindfulness meditation were not included in this study because long-term meditation has been found to positively affect cognitive performance (Moore \& Malinowski, 2009) and could not be distinguished from any effects of the interventions in this study.

5. Individuals who were had color-deficient vision were not included in this study because the Stroop task requires participants to identify the colors of words. 


\section{Chapter 2}

\section{Review of Literature}

In this work, the effects of an acute bout of moderate intensity aerobic physical exercise, mindfulness mediation neurofeedback, and mindfulness meditation on executive function as measured by Stroop task (Stroop, 1935) were examined. Enhancements to various aspects of cognitive performance have been demonstrated as a result of acute physical exercise (Chang et al., 2012), mindfulness meditation (Cahn \& Polich, 2013), and neurofeedback treatments (Gruzelier, 2014a). In this chapter, each intervention and its relationship to cognitive performance is reviewed. In the final section, how these interventions combine to affect cognitive performance is examined.

\section{Physical exercise}

The beneficial effect of a single bout of exercise, also called acute exercise, on cognitive performance has been observed and documented for several decades (Chang et al., 2012; Debray, Biswas, Biswas, Sha, \& Pal, 2015; Etnier et al., 1997; Gutin, 1973). While some empirical studies reported detrimental results or no significant results (e.g., Fery \& Ferry, 1997; Sparrow \& Wright, 1993), meta-analyses have indicated an overall small but significant positive effect of acute exercise on cognitive performance (Chang et al., 2012; Etnier et al., 1997; Lambourne \& Tomporowski, 2010; Sibley \& Etnier, 2003). Recent research on the effects of acute physical exercise on cognitive performance with previously untested populations and/or exercise types concur with the meta-analyses of the last decade (Debray et al., 2015; Barenberg, Berse, \& Dutke, 2015; Ma, Le Mare, \& Gurd, 2015). For example, Debray et al. (2015) studied the effects of an acute bout of 
step up exercise on males using the Stroop task. Barenberg et al. (2015) found that intense acute physical exercise with an ergometer cycle improved executive control. Ma et al. (2015) found that a short 4-minute bout of high-intensity interval exercise improved selective attention in children.

Etnier et al. (1997) reported that there are several physiological explanations for why acute exercise has a positive effect on cognition. First, increased cranial blood flow as a result of moderate or strenuous exercise may increase nutrients to the brain, thereby aiding in cognition (Etnier et al., 1997). Acute exercise also benefits memory as a result of increased neurotransmitter levels, including levels of norepinephrine, serotonin, endorphins, and brain-derived neurotropic factor (BDNF) (Etnier et al., 1997). Etnier et al. stated that exercise might cause positive changes in the structural makeup of the brain, as exhibited in two studies conducted with rats (Black, Isaacs, Anderson, Alcantara, \& Greenough, 1990; Isaacs, Anderson, Alcantara, Black, \& Greenough, 1992). Similarly, van Praag, Kempermann, and Gage (1999) demonstrated that physical exercise triggers the birth of new neurons in the brain of adult rodents.

Physical exercise and cognition has been a popular topic of study for special populations, especially older adults and children. Age-related cognitive decline makes studying the effects of physical exercise on older individuals especially challenging. A meta-analysis by Colcombe and Kramer (2003) found that in 18 empirical studies published between 1966 and 2001, physical exercise had the greatest effect on executive control processes such as coordination, planning, and working memory in older individuals. Studies seeking to tie improved attention to physical exercise in school 
children are understandably popular given controversies over physical education in schools. Sibley and Etnier (2003) positively linked physical activity with improved cognition in children, at an even higher effect size than found in adults in previous metaanalyses, suggesting that physical exercise may be particularly advantageous for children. Nevertheless, physical exercise has been linked to improvements in cognitive performance throughout the lifespan (Hillman, Erickson, \& Kramer, 2008).

Chang et al. (2012) analyzed the literature for studies on acute physical exercise and cognitive performance and found six moderating variables that frequently surfaced: exercise type (acute or chronic), exercise intensity (low, moderate, or strenuous), participant fitness level, exercise activity (aerobic or anaerobic), timing of cognitive task (during, immediately after, or more than one minute following exercise), and type of cognitive task. The present study extracted best practices from each of the six moderating variables studied the effects of an acute bout of moderate-intensity exercise on executive function with the Stroop task (Stroop, 1935), which was administered immediately following the exercise bout. The following subsections elaborate on the empirical and theoretical research based on the six aforementioned moderating variables.

Exercise type as a moderating variable. Studies of physical exercise and cognitive performance typically use either acute (single-bout) or chronic (long-term) exercise interventions (Etnier et al., 1997). Pérez, Padilla, Parmentier, and Andrés (2014) examined the effects of chronic exercise on attentional functions in physically active college-aged participants, who practiced aerobic activities for at least six hours a week for 10 years or more and sedentary college-aged participants, who had not participated in 
exercise for more than two hours per week in the last four years. Pérez et al.'s results indicated a significant positive effect of chronic exercise on executive function in young adults. Monleón et al. (2015) studied the effects of an eight-month physical exercise program on obese adults. Monleón et al. (2015) found that in addition to increases in cardiovascular fitness and decreased BMI, chronic exercise benefited participants' sustained attentional capabilities and reaction time. The studies by Pérez et al. (2014) and Monleón et al. (2015) are in contrast to previous studies conducted on chronic exercise and executive function that did not find positive significant effects (e.g. Boucard et al., 2013). Pérez et al. (2014) hypothesized that previous studies did not indicate positive effects of chronic exercise on cognitive performance in young adults because participant selection criteria may have been too homogeneous, executive functions are difficult to measure due to low reliability and their multifaceted nature, and the cognitive function of young adults is difficult to measure because they are at their peak performance.

Exercise intensity as a moderating variable. The primary framework for understanding the effects of exercise intensity is the inverted-U hypothesis (Davey 1973), based off of Yerkes and Dodson's (1908) arousal-performance theory. The Yerkes and Dodson principle positions performance in relation to arousal where moderate levels of arousal are assumed to result in better performance then at the extremes of low and high arousal levels. While firm evidence of a an inverted-U relationship between acute exercise and cognitive performance has yet to be established, meta-analyses by Etnier et al. (1997) and Lambourne and Tomporowski (2010) found small but significant effects in 
favor of the inverted-U hypothesis. Similarly, a meta-analysis by McMorris and Hale (2012) investigated the relationship between intensity of acute exercise and cognitive speed and accuracy. McMorris and Hale found that moderate intensity exercise, as opposed to high or low-intensity exercise, increased cognitive processing speed, adding support to the inverted-U hypothesis. Individual empirical studies also found results in favor of an inverted- $U$ relationship between acute exercise intensity and cognitive performance (e.g., Kamijo et al., 2004; Kamijo, Nishihira, Higashiura, \& Kuroiwa, 2007). In contrast, a meta-analysis by Chang et al. (2012) indicated that intensity does not affect cognitive performance when performance is measured during exercise. Therefore, Chang et al.'s findings failed to support an inverted-U hypothesis when cognitive performance is required during exercise.

One reason why studies report different findings with regard to exercise intensity effects on cognitive performance may be that these studies neglected to account for participant fitness levels. A recent study by Huttermann and Memmert (2014) found that participant fitness level may impact the effects of exercise intensity on cognitive performance. Huttermann and Memmert tested the attentional capabilities of athletes and non-athletes at varying levels of exercise intensity. They found that while an inverted-U was found for non-athletes, attentional capabilities continued to increase for athletes even during strenuous physical activity. Therefore, the relationship of an inverted-U merits further study, especially in the presence of other moderating variables such as cognitive task timing and participants' fitness levels. 
Participant fitness level as a moderating variable. As Huttermann and Memmert (2014) demonstrated, fitness level is a primary moderator producing an interaction between exercise and attentional capabilities. Chang et al. (2012) observed that for cognitive tasks administered directly following exercise, low and above average fitness level participants experienced a significant positive effect on cognitive performance, while there was no significant difference for participants of average fitness level. The present study sought to add to this body of knowledge by comparing pre-study fitness levels based on American College of Sports Medicine (ACSM) guidelines (2014) with Stroop task performance to assess any relationship between overall fitness level and participants' boost in cognitive performance after an acute bout of moderate intensity physical exercise.

Participant fitness levels may also have a physiological effect that directly contributes to the effect of an acute physical exercise bout on cognitive performance. Brain-derived neurotropic factor is a secreted protein that supports neuron survival and growth and has been shown to increase with physical exercise (Neeper, Gómez-Pinilla, Choi, \& Cotman, 1995).

Zoladz et al. (2008) examined brain-derived neurotropic factor levels after a fiveweek cycle-based endurance training program with young, healthy males. Post-exercise and at-rest levels of brain-derived neurotropic factor climbed during the five weeks of training, suggesting that participant fitness level may have an effect on brain-derived neurotropic factor levels (Zoladz et al., 2008). Nofuji et al. (2012) examined brainderived neurotropic factor levels in physically active and sedentary female participants 
after undergoing exercise conditions with three different intensities: low, moderate, and strenuous. For the strenuous condition, sedentary participants saw a return to brainderived neurotropic factor baseline levels during the recovery period (Nofuji et al., 2012). Physically active participants, however, experienced elevated brain-derived neurotropic factorlevels directly following exercise and then a dip below baseline during the recovery period (Nofuji et al., 2012). Nofuji et al. hypothesized that physically active participants may take advantage of the secreted brain-derived neurotropic factor for muscle repair following strenuous exercise. From a participant fitness perspective, the findings of Zoladz et al. (2008) and Nofuji et al. (2012) demonstrate that preexisting fitness level affects brain-derived neurotropic factor production and, as a result, possibly cognitive performance, although evidence of such has yet to be established.

Exercise activity as a moderating variable. Aerobic exercise uses oxygen to produce energy. Aerobic exercise includes exercises that are typically of moderateintensity and sustained over time, such as cycling or jogging. Colcombe and Kramer (2003) found that aerobic exercise had positive effects on cognitive function on a cellular level in animals. Anaerobic exercise does not require oxygen to produce energy. Anaerobic exercise builds strength through bursts of strenuous activity, such as in weightlifting. Although Chang et al. (2012) stipulated that more research is necessary, they reported that anaerobic exercise may have negative effects on cognitive performance. However, combined anaerobic-aerobic exercise produced larger cognitive performance effect sizes than aerobic exercise alone (Chang et al., 2012). 
The majority of studies conducted on physical exercise and cognitive performance include aerobic activities (Lambourne \& Tomporowski, 2010). Lambourne and Tomporowski asserted that even within aerobic activities, the type of exercise has an effect on cognitive performance. For example, treadmill running requires a greater level of attention than stationary cycling because treadmill participants must cognitively attend to balance and pace in order to successfully run on the machine (Lambourne \& Tomporowski, 2010). Therefore, it seems that aerobic exercise that requires little attentional focus may have the largest positive effect on cognitive performance if the cognitive task is administered during exercise.

Timing of cognitive task as a moderating variable. In a now widely accepted theory of attention, Kahneman (1973) stated that attentional resources are limited, and that that limitation may restrict an individual's cognitive performance when resources are spread across several foci. As such, when cognitive tasks are administered during exercise the effects may be negative because attentional resources are split between the exercise and the cognitive tasks (Brisswalter, Collardeau, \& Rene, 2002; Lambourne \& Tomporowski, 2010).

In contrast, Chang et al. (2012) found fluctuation in cognitive performance effect size when cognitive tasks were administered during exercise. Cognitive performance effects in minutes 0-10 were not significant, effects in minutes 11-20 were negative, and effects after 20 minutes were positive (Chang et al., 2012). In this regard, Chang et al. and Brisswalter et al. (2002) agreed that at least 20 minutes of aerobic exercise is needed 
to observe positive effects on cognitive performance, whether the cognitive task is administered during or following exercise.

Type of cognitive task as a moderating variable. A wide variety of cognitive tasks have been studied in connection with acute physical exercise including attention (Debray et al., 2015; Kubesch et al., 2009; Ma et al., 2015), executive function (Barenberg et al., 2015; Chang et al., 2014), memory (Ferris, Williams \& Shen, 2007), and information processing and reaction time (Davranche, Burle, Audiffren, \& Hasbroucq, 2005). A meta-analysis by Chang et al. (2012) of 79 studies found that acute physical exercise had positive effects on cognitive tasks of executive function and crystalized intelligence. A meta-analysis by Tomporowski (2003) found that acute exercise benefitted information processing and long-term memory. The present study examined cognitive performance, specifically executive function, with the Stroop task (Stroop, 1935). Significant positive effects have been observed in empirical studies including the Stroop task (e.g. Barella et al., 2010; Chang et al., 2014; Labelle et al., 2013).

In sum, various effects of physical exercise on cognitive function have been observed on physiological and performance levels. Moderating variables, including exercise type, exercise intensity, participant fitness level, exercise activity, cognitive task timing, and type of cognitive task contribute to or detract from cognitive performance depending on the parameters used within each variable (Chang et al., 2012; Etnier et al., 1997; McMorris and Hale, 2012; Tomporowski, 2003). As such, in this work the study design was inclusive of what was learned from the findings demonstrated in previous 
empirical studies so as to carefully investigate the most optimum environment for cognitive performance boost across the three interventions.

\section{Mindfulness meditation}

Mindfulness is a state of being in which an individual is aware of and attentive to what is going on in the present moment without passing judgment on what is happening (Kabat-Zinn, 1990). The state of mindfulness is achieved by undergoing mindfulness meditation, a mental practice in which an individual maintains a calm, non-judgmental mind while focusing on sensations of the body and breath (Wallace \& Shapiro, 2006). Mindfulness meditation has been linked to improved cognitive performance in both healthy (Cahn \& Polich, 2013; Eberth et al., 2012) and clinical populations (Grossman, Niemann, Schmidt, \& Walach, 2004).

Kabat-Zinn, the creator of the popular clinical framework the mindfulness-based stress reduction program outlined the process for meditation in his book Full Catastrophe Living: Using the Wisdom of Your Body and Mind to Face Stress, Pain, and Illness. Participants are encouraged to set aside a specific time and place for meditation (KabatZinn, 1990, p. 61). During a mindfulness meditation session, participants sit on the floor or in a chair, assuming an "alert and relaxed body posture," aligning the back, shoulders, and neck and relaxing the hands (Kabat-Zinn, 1990, p. 62). A participant's attention then goes to the in-and-out motion of the breath, focusing on the sensations of the breath in the body (Kabat-Zinn, 1990, p. 62). When the mind wanders, as it is bound to do no matter how committed the participant is to meditation, Kabat-Zinn instructed participants to 
observe the wandering without dwelling on or judging it, then gently bring the attention back to the breath (1990, p. 64).

The present study aimed to understand how cognitive performance, specifically executive function, may be enhanced in healthy individuals, whether by mindfulness meditation, mindfulness meditation neurofeedback, or acute physical exercise. The previous section reviewed acute physical exercise literature. The following subsections review mindfulness meditation from a historical perspective, explore the practical applications of mindfulness meditation, and review its effects on cognitive performance. While the present study was not an attempt to understand mindfulness meditation from a Buddhist perspective, Buddhist foundations are briefly discussed here to provide historical perspective. Similarly, it was not the goal of this study to explore any clinical treatment, although the effects of mindfulness mediation on clinical populations are briefly discussed to provide context as to the wide-ranging effects of the intervention.

Historical background. Western psychological methods related to mindfulness meditation draw heavily from Buddhist traditions (Sedlmeier et al., 2012). Buddhism has evolved and spread considerably in the 2,500 years since its inception, yet all practices of Buddhism are concerned with spiritual freedom and insight (Wallace \& Shapiro, 2006). Through mindfulness meditation, Buddhist meditators seek to enhance qualities such as calmness, concentration, and introspective sensitivity (Walsh, 1982). Increased concentration and introspective sensitivity lead to enhanced attention, which may be used at will as a means for personal knowledge and self-examination (Wallace, 1999). 
In 1982, Walsh noted the difficulty of studying meditation with Western tools, likening the Western investigation of mindfulness meditation to assessing the effects of psychoanalysis by measuring a patient's galvanic skin response for a short period of time (Walsh, 1982). In 2014, Kudesia and Nyima, a behavioral scientist and a Buddhist monk, argued a similar lack of context in investigations into Buddhist meditation practices (Kudesia \& Nyima, 2014). Kudesia and Nyima stated that meditation must be studied as a whole, not broken down into individual elements such as attention. While it was not the intention of the present study to discount any aspect of more holistic studies of mindfulness meditation, we believe that it is still useful to research individual parts of meditation to more fully understand the whole. Just as basic research seeks to understand a foundational element of a phenomenon as a way to understand the whole, there is a need to determine the individual elements that make up mindfulness meditation and to use those elements to advance ecologically valid applications that improve the lives of healthy and clinical populations.

Practical applications of mindfulness meditation. Although mindfulness meditation is rooted in Buddhist and Indian spiritual traditions that may aid and benefit long-term meditators, it is not necessary to fully understand the philosophy behind the process to practice and benefit from mindfulness meditation (Chow, 2014). Kristeller and Rikhye (2008) suggested that mindfulness practice releases an individual from the constant cycle of attentional scanning, ruminating, and reacting. Similarly, Slagter, Davidson, and Lutz (2011) asserted that meditation assists with the development of three regulatory skills: distraction monitoring, disengagement, and redirection. Therefore, 
mindfulness meditation is likely to help suppress undesirable cognitive behaviors while building desirable cognitive skills. As a result, Sedlmeier et al. (2012) suggested that researchers should always expect positive results when studying cognitive changes in mindfulness meditation practitioners.

Meditation has become a popular therapeutic tool in clinical settings (Grossman et al., 2004). Meditative techniques are used both alone and as an accompaniment to other medical or psychological treatments (Moore \& Malinowski, 2009). The most popular clinical meditative approach is the mindfulness-based stress reduction program developed by Kabat-Zinn (1982), which has consistently shown strong post-intervention effects across a variety of chronic disorders, including cancer, chronic pain, coronary artery disease, and fibromyalgia (Grossman et al., 2004). It was beyond the scope of this work to examine mindfulness meditation from a clinical perspective. The goal of this work was to investigate the effects of mindfulness meditation, mindfulness mediation neurofeedback, and acute physical exercise on cognitive performance with healthy individuals. For meta-analyses of health benefits related to the mindfulness-based stress reduction program, see Grossman et al. (2004) and Chisea and Serretti (2009).

Mindfulness meditation from a neuropsychological perspective has also garnered interest due to advanced neuroimaging devices such as functional MRI (fMRI), position emission tomography (PET) scans, and EEG (Cahn \& Polich, 2013). A review and metaanalysis conducted by Fox et al. (2014) indicated that while causal effects of meditation and brain structure have yet to be established, there are consistent correlational findings that suggest mindfulness meditation has positive effects on brain anatomy. Furthermore, 
a meta-analysis by Tomasino, Fregona, Skrap, and Fabbro (2013) found that neuroimaging studies indicate that default mode network, the region of the brain responsible for self-referential processing, is significantly reduced in experienced meditators when compared to non-meditators.

It is at the intersection of clinical and neuropsychological perspectives that researchers may find the most insight into the effects of mindfulness meditation. Research by Wallace and Shapiro (2006) provided a model for blending Buddhist spiritual traditions with contemporary Western psychology by proposing four types of mental balance that work together to achieve overall psychological well-being: conative (intention and motivation toward a goal), attentional (sustained, voluntary attention), cognitive (calm, non-judgmental presence in a moment), and affective (freedom from excessive emotions as a result of conative, attentional, and cognitive balance). From the Buddhist perspective, a meditator seeks to enhance these qualities in order to alter perspective to the extent that enlightenment is obtained, thereby ceasing personal suffering (Kudesia \& Nyima, 2014). From a Western psychological perspective, positive psychology encourages the cultivation of goal setting, positive attitude, and internal mental training to support overall well-being (Seligman, 2006). Thus, there is considerable overlap between the Buddhist and psychological perspectives that may aid in advancing the understanding of attention-based mental training and overall well-being.

Mindfulness meditation and cognitive performance. Wallace and Shapiro (2006) included attention as one of the four types of mental balance, noting that attention is a core element of Buddhist traditions as well as Western psychology. Buddhist 
philosophies state that all humans, not just those with conditions such as ADHD, are predisposed to inattention, and that inattention causes issues in everyday life (Wallace \& Shapiro, 2006). Within Western psychology, clinical and neuropsychological descriptions of mindfulness contain attention training as a central element (Moore \& Malinowski, 2009). For example, Kabat-Zinn, the creator of the mindfulness-based stress reduction program, instructs meditators to:

Give full attention to the feeling of the breath as it comes in and full attention to the feeling of the breath as it goes out ... and whenever we find that our attention has moved elsewhere, wherever that may be, we just note it and let it go and gently escort our attention back to the breath. (Kabat-Zinn, 1990, p. 64-65) Furthermore, attention training is common across different types of meditation (Davidson \& Goleman, 1977). Two styles of meditation exist based on how attention is focused, termed open monitoring mediation and focused attention meditation, although in practice these two styles are often used in conjunction to achieve the desired effects (Cahn \& Polich, 2013; Sedlmeier et al., 2012). Open monitoring practice encourages the meditator to become an impartial third-party observer to thoughts, feelings, and sensations that arise (Cahn \& Polich, 2013; Sedlmeier et al., 2012). Focused attention practice places focus on a specific object, such as breath, an image, or mantra (Cahn \& Polich, 2013; Sedlmeier et al., 2012). The present study contained elements of both open monitoring and attention practice meditation. During the pre-session introduction to Muse the user is instructed to count breaths as well as gently and nonjudgmentally bring attention back to the breath when the mind wanders. In the present study, both the mindfulness meditation and the mindfulness meditation neurofeedback groups received the pre-session introduction. 
The Stroop task is a well established measure of executive function that tests attention, cognitive flexibility, inhibition, and short-term memory, several of the same skills that mindfulness meditation enhances (Chan \& Woollcott, 2007; Moore \& Malinowski, 2009; Wenk-Sormaz, 2005). Of Wallace and Shapiro's (2006) four types of mental balance (conative, attentional, cognitive, and affective), the Stroop task evaluates two: attention and cognition. This makes the Stroop task well suited to test how mindfulness meditation affects executive function.

In practice, Moore and Malinowski (2009) used the Stroop task to investigate the cognitive flexibility and attentional capabilities of experienced and naïve meditators. Moore and Malinowski found that experienced meditators performed significantly better than the naïve group on all measures of attention. Similarly, Wenk-Sormaz (2005) investigated the selective attention processes cultivated by mindfulness meditation with the Stroop interference task and found that those who received the mindfulness meditation treatment performed significantly better on the Stroop task than those who received other attention-based treatments. Chan and Woollacott (2007) tested executive attentional capabilities of long-term meditators with the Stroop task and found that meditators performed significantly better on the Stroop task when compared to a control group, suggesting that meditation has a positive effect on Stroop task performance.

In conclusion, there is currently no formal psychological theory as to the effects of mindfulness meditation (Sedlmeier et al., 2013). However, studies that include extensive mindfulness training or experienced meditators have found that mindfulness meditation improves aspects of cognitive performance (Chan \& Woollacott, 2007; Moore 
\& Malinowski, 2009), and overall well-being (Brown \& Ryan, 2003). It also may have a positive impact on physiological brain structure (Cahn \& Polich, 2013; Fox et al., 2014; Tomasino et al., 2013). Mindfulness meditation studies have so far included more than one treatment session, however, research findings support that even a single session of neurofeedback can have significant results (Kluetsch et al., 2014; Ros et al., 2013).

\section{Neurofeedback}

Neurofeedback is an operant conditioning paradigm in which participants' brainwaves are read by an EEG or similar device and relayed back in the form of audio and visual feedback (Vernon, 2005). Neurofeedback is similar to better-known biofeedback, which provides feedback on an individual's physiological functions, such as heart rate, so that the individual may learn to control the function at will. A metaanalysis by Gruzelier (2014a) indicated that neurofeedback has a significant positive effect on cognitive performance.

The present study used Muse, a brainwave-sensing headband produced by InteraXon Inc., to provide neurofeedback to participants. Muse senses brainwave levels, active, neutral, or calm, and provides audio and visual feedback to a participant's mobile device. As the participant learns to associate certain cerebral sensations with the neurofeedback, control may be achieved over time (Vernon, 2005). To achieve calm brainwaves, Muse instructs participants to focus attention on the breath, the core activity of mindfulness meditation. Therefore, Muse provides mindfulness meditation neurofeedback. 
The present study investigated how three interventions, acute physical exercise, mindfulness meditation, and mindfulness meditation neurofeedback, effect cognitive performance. In the previous two sections literature on physical exercise and mindfulness meditation is investigated. In the following subsections an investigation of the literature on historical background of neurofeedback procedures and the connection of neurofeedback to cognitive performance are explored.

Historical background. Research by Kamiya $(1968,1969)$ first demonstrated the ability of healthy individuals to regulate electrical activity in the brain as a result of operant conditioning, a process known today as neurofeedback. As an operant conditioning paradigm, neurofeedback training teaches a participant to use the feedback from an EEG or other brainwave-monitoring device to control the brain's electrical activity (Egner \& Gruzelier, 2001).

Flawed empirical studies and exaggerations about neurofeedback's capabilities in clinical populations in the 1960s and early 1970s resulted in a steep decline of neurofeedback research in the United States in the 1970s and 1980s (Gruzelier, 2014a). Research conducted by Sterman (e.g., Sterman, Howe, \& MacDonald 1970; Sterman, MacDonald, \& Stone, 1974; Sterman \& Wyrwicka, 1967) and Lubar (e.g., Lubar \& Lubar, 1984; Lubar \& Shouse, 1976) primarily kept the study of neurofeedback alive until recently. Recent popularity in Internet-based brain training applications such as Lumosity (www.lumosity.com), FitBrains (www.fitbrains.com), and Brain Metrix (www.brainmetrix.com) that aim to improve cognitive function and attentional capabilities have caused a renewed interest in neurofeedback research (Rabipour \& Raz, 
2012). As brain monitoring devices and brain training applications become more used by the general population to enhance cognitive performance, it is increasingly imperative to investigate the scientific basis, or lack thereof, of such devices (Rabipour \& Raz, 2012).

Neurofeedback procedures. The goal of neurofeedback training is to teach an individual to distinguish different types of cortical activity by exposing the individual to different types of brainwaves and providing feedback so that the sensations of the brainwave types may be learned (Vernon, 2005). At first, neurofeedback participants may be unable to discern what causes the changes in feedback (Wang \& Hsieh, 2013). However, through gradual practice and trial and error, most participants develop strategies to access and alter various brainwave frequencies (Wang \& Hsieh, 2013).

During a traditional neurofeedback session, EEG sensors are placed on the participant's scalp and the resulting electrical readings are instantaneously shown to the participant in the form of audio, visual, or audio-visual feedback (Vernon, 2005). Instantaneous feedback is critical for the participant to associate specific sensations with the EEG feedback cue.

Another intervention used in a neurofeedback session has been to "gamify" the application as a means to motivate participants to continue engaging even after becoming fatigued (Vernon, 2005). In gamified neurofeedback, a participant receives audio-visual feedback and a reward, usually in the form of points, when a predefined goal is reached, with the goal being to accumulate as many points as possible (Vernon, 2005). Participant compensation may or may not be tied to the number of points received from participants' 
neurofeedback work. Over time, brainwave self-control can be learned and may be accessed even without immediate biofeedback (Wang \& Hsieh, 2013).

A number of different neurofeedback protocols have been used throughout the decades in an attempt to produce peak cognitive performance (Gruzelier, 2014a). In traditional EEG neurofeedback, specific brainwave frequencies are enhanced or suppressed to produce the desired effect (Gruzelier, 2014a). For example, theta $(4-7 \mathrm{~Hz})$ has been connected to working memory and alpha (7-12 Hz) with semantic long-term memory (Kilmesch, 1999). For a review of such associations the work done to map specific EEG frequencies to cognitive outcomes see Kilmesch (1999).

Gruzelier (2014b) reported that in addition to varying protocols, the number of neurofeedback sessions change between empirical studies, ranging from one to thirty-five sessions with healthy participants. Most investigators find eight to ten sessions successful, although cognitive gains have been observed in as few as one session (Gruzelier, 2014b; e.g., Kluetsch et al., 2014; e.g. Ros et al., 2013).

Ros et al. (2013) conducted one such single-session neurofeedback experiment and observed causality between a 30-minute session of alpha rhythm $(8-12 \mathrm{~Hz})$ suppression neurofeedback and decreased mind wandering when compared to a placebo group who received inaccurate, prerecorded neurofeedback. Evidence was provided by fMRI neuroimages showing additional resting-state neural plastic reinforcement and connectivity in neurofeedback participants in the area of the brain that seems to be responsible for cognitive control (Ros et al., 2013). 
Another successful single-session neurofeedback study was conducted by Kluetsch et al. (2014) in which individuals suffering from childhood abuse-related PTSD underwent a 30-minute session of alpha rhythm suppression neurofeedback training before being scanned by an fMRI. Post-neurofeedback fMRI neuroimages demonstrated that participants experienced increased resting-state neural connectivity and a rebound of alpha synchronization, resulting in subjective calmness and feelings of well-being (Kleutsch et al., 2014).

Neurofeedback and cognitive performance. While there are no studies known to the author that combine neurofeedback, mindfulness meditation, and tests of cognitive performance, there has been research on neurofeedback and cognitive performance, specifically executive function. Neurofeedback and executive function research is most often conducted with individuals who have ADHD because ADHD contributes to difficulty focusing attention and low inhibition functions (Bink et al., 2014). Bink et al. (2014) conducted a randomized control study in which participants with ADHD underwent neurofeedback intervention, the effects of which were tested on several cognitive performance tests, including the Stroop task. Positive significant results were found on the Stroop task for participants who underwent neurofeedback, suggesting that neurofeedback has an impact on Stroop task performance (Bink et al., 2014).

In sum, the literature supports the use of neurofeedback as a non-invasive tool used to enhance cognitive performance in healthy (Gruzelier, 2014a) and clinical populations (e.g. Kluetsch et al., 2014; Sterman et al., 1974). While studies including a single session of neurofeedback are somewhat rare, Kleutsch et al. (2014) and Ros et al. 
(2013) conducted two such studies and found significant effects when only one session was used.

\section{Mindfulness meditation neurofeedback}

There have not yet been any empirical studies conducted that investigated mindfulness meditation neurofeedback. Lai et al. (2014) compared the attentional effects of a single bout of mindfulness meditation and prefrontal hemoencephalography (FpHEG), a type of neurofeedback, on healthy participants. Lai et al. found that Fp-HEG neurofeedback participants demonstrated improved attentional capabilities, while the mindfulness meditation group did not differ from the control. A master's thesis by Chow (2014) compared a single session of mindfulness meditation to EEG-Alpha wave neurofeedback. Chow found that neurofeedback and mindfulness meditation group participants exhibited reduced EEG-Alpha event-related desynchronization, suggesting that they required less cognitive effort to complete the Stroop task. Neither mindfulness meditation nor neurofeedback groups exhibited improvements in Stroop task performance, however, unlike the present study, Lai et al.'s and Chow's research did not study the combined effects of mindfulness meditation and neurofeedback, suggesting that future research is needed in this area.

Brandmeyer and Delorme (2013) explored the histories of mindfulness meditation and neurofeedback as interventions, discussed similarities between and benefits of the interventions, and call for mobile neurofeedback systems that aid in meditation and are based in scientific research. In the present study, I aimed to add to this body of knowledge now that a mobile mindfulness meditation neurofeedback device exists. 
Chittaro and Vianello (2014) examined a mobile application that monitors thought distancing through mindfulness meditation in comparison with two non-computer-based thought distancing methods with meditation naïve participants. Chittaro and Vianello found that the mobile application performed better in terms of achieved mindfulness, perceived difficulty, and pleasantness. This is an encouraging first step in combining mindfulness mediation with technological aids.

Another recent study conducted by Vidyarthi and Riecke (2014) combined mindfulness meditation with current technology. Vidyarthi and Riecke investigated the Sonic Cradle, a suspended, hammock-like chair placed in a dark room with piped-in audio that encouraged participants to focus on the breath. After a 15-minute session in the chamber, participants reported sensations similar to mindfulness including calmness and mental clarity (Vidyarthi \& Riecke; 2014).

As technology enables us to investigate further into the subjective and physiological effects of mindfulness meditation and neurofeedback, it seems a natural progression to study the combination of these similar tools in order to enhance cognitive performance.

\section{Summary of the Review of Literature}

Acute physical exercise (Chang et al., 2012), mindfulness meditation (Cahn \& Polich, 2013), and neurofeedback (Gruzelier, 2014a) have independently been found to have positive effects on cognitive performance, and, more specifically executive function. The Stroop task is a one of the three most frequently used tests of executive 
function (Etnier \& Change, 2009), and has been found to be sensitive to the effects of acute physical exercise (Chang et al., 2014), mindfulness meditation (Deepeshwar et al., 2015), and neurofeedback (Bink et al., 2014) independently.

Few studies have so far investigated the effects of mindfulness meditation and neurofeedback in the same study (Chow, 2014; Lai et al., 2014). No studies have so far investigated the acute physical exercise, mindfulness meditation, and neurofeedback in a single study, nor the combined intervention of mindfulness meditation neurofeedback. The goal of the present study was to fill that gap in the literature by examining the effects of three interventions on Stroop task performance. 


\section{Chapter 3}

Method

The purpose of this study was to compare the effects of mindfulness meditation neurofeedback, mindfulness meditation, and acute aerobic bout on executive function. A between-subjects design with three conditions was used. Participants engaged in one of three randomly assigned conditions: acute physical exercise, mindfulness meditation neurofeedback, or mindfulness meditation. Executive function was measured by Stroop task time and Stroop task errors.

The mindfulness meditation group is useful for two reasons. First, because mindfulness meditation is known to positively affect cognitive performance (Cahn \& Polich, 2013), it provides a baseline of data with which to compare the untested mindfulness meditation neurofeedback condition. The method of implementation of the mindfulness meditation intervention will help study expectancy that participants may experience by merely wearing an external device such as the Muse.

For the exercise group, self-monitored moderate-intensity exercise was selected to improve ecological validity of the treatment and maximize the potential for cognitive improvement. Research has demonstrated that 20 minutes of acute, moderate-intensity exercise provides the largest gains in cognitive performance compared to other forms and durations of exercise (Chang et al., 2012). Since moderate intensity may vary by individual, it is important to customize target heart rate based on the participant's age and then to encourage participants to self-monitor heart rate to stay within range of the customized heart rate. 
For the mindfulness meditation neurofeedback and mindfulness meditation groups, participants engaged in 20 minutes of mindfulness meditation neurofeedback or mindfulness meditation, respectively, to standardize the amount of time spent in the intervention. Although traditional mindfulness meditation practices start beginners at 510 minutes of practice (Kabat-Zinn, 1990), Ros et al. (2013) observed positive results after a single 30-minute session of neurofeedback, suggesting that more time may be needed in combined mindfulness meditation neurofeedback to observe results. The following section provides information about the study participants, measures, and procedures, design, and analysis.

\section{Design and Analysis of Data}

To examine the relationship of each of the three interventions, acute physical exercise, mindfulness meditation neurofeedback, and mindfulness meditation and Stroop performance, this study used a between-subjects design. Participants were randomly assigned to one of the three intervention groups. Stroop task performance was measured in two ways: total time the participant needed to complete 50 trials of the Stroop task, and total number of errors the participant committed over the 50 trials. Differences between intervention groups were then tested by two one-way ANOVAs, one for Stroop task time and one for Stroop task number of errors.

\section{Participants}

The participants were 42 healthy adults recruited for this study from introductory psychology classes and advertisements placed around a large university. A power analysis using G*Power (Faul, Erdfelder, Lang, \& Buchner, 2007) indicated that the 
minimum number of participants needed for a medium effect size $(\alpha=.05$, power $=.80)$ was 160 . However, resource and time limitations required this research to be conducted on a smaller scale. Previous research on physical exercise and cognitive performance found effects that were small (Cohen, 1988), but positive and consistent across studies (Etnier et al. 1997; Chang et al., 2012). If this research were to result in similar effect sizes despite the small sample size, it would indicate further similar studies are warranted.

To qualify for this study, participants had to be between the ages of 18 and 69 years and healthy enough to perform a bout of moderate-intensity aerobic exercise. Participants were screened for participation using the Physical Activity Readiness Questionnaire (PAR-Q; See Appendix A), which determined readiness to participate in exercise by identifying potential health issues that could limit an individual's ability to exercise safely. Participants were also screened for inclusion with a Pre-Experiment Questionnaire (see Appendix B), which included questions on participant physical activity level and mindfulness meditation practices. Participants were required to meet or exceed the American College of Sports Medicine's (ACSM, 2014) aerobic exercise frequency and intensity recommendations in order to participate in this study. The ACSM recommends 150 minutes or more moderate intensity exercise per week or 75 minutes or more of high intensity exercise per week (ACSM, 2014). This delimitation was in place to ensure that participants were accustomed to regular exercise and fit enough to participate in exercise without injury (ACSM, 2014). Individuals who practiced mindfulness meditation were not included in this study because long-term 
meditation positively affects cognitive performance (Moore \& Malinowski, 2009) and could not be distinguished from any affects of the interventions in this study. Because the Stroop task requires participants to identify the color of the word, participants could not be colorblind.

Participants who answered negatively to all questions on the PAR-Q, met the physical exercise requirements, did not engage in mindfulness meditation, and were not colorblind were randomly assigned into one of three groups by drawing lots. Participants who answered positively to at least one question on the PAR-Q, did not meet the physical exercise requirements, practiced mindfulness meditation, or were colorblind were not included in this study. In total, twelve participants were excluded from the current study. Nine individuals were excluded from this study due to not meeting the minimum exercise requirements, two individuals were excluded due to colorblindness, and one individual was excluded due to regular meditation practice. All participants were required to read and sign informed consent (see Appendix C) as approved by the San José State University Institutional Review Board (see Appendix D).

\section{Measures}

The Physical Activity Readiness Questionnaire. The PAR-Q (see Appendix A) was administered to all participants in the acute exercise group prior to exercising to ensure that they were healthy enough to participate in physical exercise. Participants had to answer negatively to all questions on the PAR-Q to be included in the study. 
Heart rate reserve equation. A moderate-intensity bout of aerobic exercise was used in the physical exercise condition. Moderate-intensity was individualized based on participant age and using the heart rate reserve (HRR) equation. HRR is the difference between an individual's maximum heart rate $\left(\mathrm{HR}_{\max }\right)$ and resting heart rate $\left(\mathrm{HR}_{\mathrm{rest}}\right)$, multiplied by a relative heart rate intensity percentage: $H R R=$ intensity $\%\left(H R_{\max }-\right.$ $\left.H_{R_{\text {rest }}}\right)+\mathrm{HR}_{\text {rest }}$ Maximum heart rate was determined for each participant with Tanaka et al.'s (2001) equation: $\mathrm{HR}_{\max }=208-(0.7 \mathrm{x}$ age $)$. Cited in the ACSM's Guidelines for Exercise Testing and Prescription (2014; p. 168) Tanaka et al.'s (2001) equation is recommended for determining maximal heart rate healthy men and women. The participant's resting heart rate was collected after five minutes of rest, before he or she began any physical activity. A relative heart rate intensity of 55\% was used when calculating HRR, as the ACSM categorizes $40 \%<\mathrm{HRR}<60 \%$ as moderate intensity (2014, p. 165). For example, for a 20-year-old person with an average maximum heart rate of 194 beats per minute (BPM) and a resting heart rate of $65 \mathrm{BPM}$, using 55\% relative heart rate intensity, $\mathrm{HRR}=0.55(194-60)+60$ (Tanaka et al., 2001). Therefore, the example HRR = 134 BPM.

Stroop task. The Stroop task (Stroop, 1935) was designed to measure attention, cognitive flexibility, inhibition, and short-term memory components of cognitive performance. Words are presented to participants one at a time in different colored ink or pixels. The word spells out blue, green, red, or yellow, but the color of the word does not match what the word spells. A correct answer is one in which the participant says the 
color of the word, not what the word spells. For example, the word yellow was written in green pixels and the participant must say "green."

Prior to the implementation of the procedures, participants were instructed to respond as quickly and accurately as possible. Each word was spelled out individually in 80-point Times New Roman font in the center of a Power Point slide with white background (see Figure 1). When presented, the letters displayed between $1 \mathrm{~cm}$ and 1.5 $\mathrm{cm}$ tall on the laptop screen. The slides were displayed on a 13-inch laptop screen with screen resolution $1280 x 800$ pixels. Participants sat approximately 18 inches from the laptop screen. The researcher sat next to the participant and advanced the slides as the participant answered correctly. For incorrect answers, the researcher said "try again" and the participant had to verbally correct the error before the researcher advanced to the next slide. When an error was made, the researcher also pressed the button on a handheld tally counter. Stroop task time was calculated manually from the time the researcher showed the first Stroop task trial slide to the participant and ended after the participant spoke the correct answer following the fiftieth trial slide. The researcher, for post-experiment statistical analysis, recorded total time and number of errors. Time was measured by the researcher in hundredths of seconds using the Stopwatch application by Tim O's Studios version 4.0 for iOS. 


\section{Yellow}

Figure 1. Illustrated is an example of a Stroop task trial slide.

\section{Instrumentation}

Stationary bicycle. A Marcy upright stationary bicycle with magnetic resistance, model ME-708 (see Figure 2), was used. Participants in the acute exercise group performed a 20-minute bout of moderate intensity exercise using the stationary bicycle.

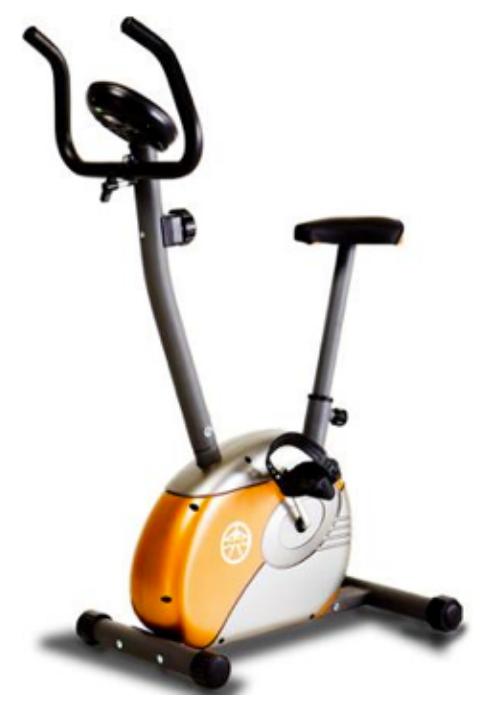

Figure 2. Represented is an image of the Marcy ME-708 stationary bicycle that was used for the acute physical exercise group. 
Heart rate monitor. Heart rate was assessed using the MIO 0021US-BLK2 Drive+ Strapless Heart Rate Monitor Watch (MIO Global, Vancouver, British Columbia). The heart rate monitor (see Figure 3) was used to gather participant resting heart rate before beginning exercise. Once positioned on the stationary bicycle and after a 5-minute warm-up, participants were asked to self-monitor heart rate $\pm 5 \mathrm{BPM}$ of their HRR for 20 minutes using the heart rate monitor. After exercise, a 2.5-minute cool-down allowed the participant's heart rate to return to normal before beginning the Stroop task.

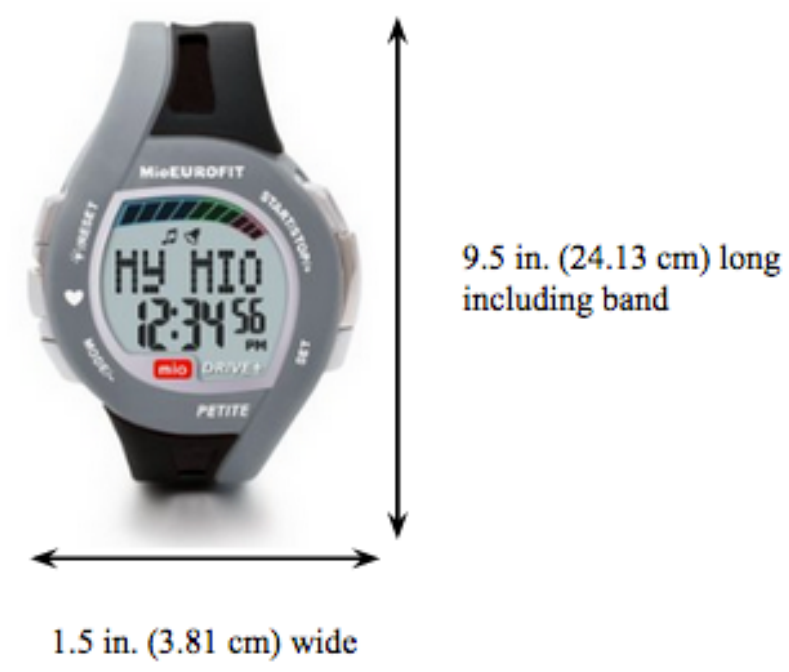

Figure 3. Represented is the MIO 0021US-BLK2 Drive+ Strapless Heart Rate Monitor Watch that was used to monitor heart rate for the acute physical exercise group participants.

Muse brain-sensing headband. Muse is a brainwave-sensing headband created by InteraXon, Inc. that is marketed to consumers as a way to improve focus, attention, and composure (Muse by InteraXon Inc., 2015). The headband weighs 2oz. and contains seven dry sensors, five across the forehead and two behind the ears that detect electrical frequencies in the scalp (see Figure 4). Muse measures the presence or absence of five 
brainwaves: alpha, beta, gamma, delta, and theta, and reports back a participant's overall brainwave activity as either active, neutral, or calm via the Muse Calm application (Muse Calm, 2014). The Muse Calm application delivers neurofeedback to the participant in the form of visual changes on the device's screen and changes in sound as the participant's brainwaves become more active or calmer. The device is not capable of changing the wearer's brainwave patterns, reading his or her thoughts, or helping the wearer to move things using only thoughts.

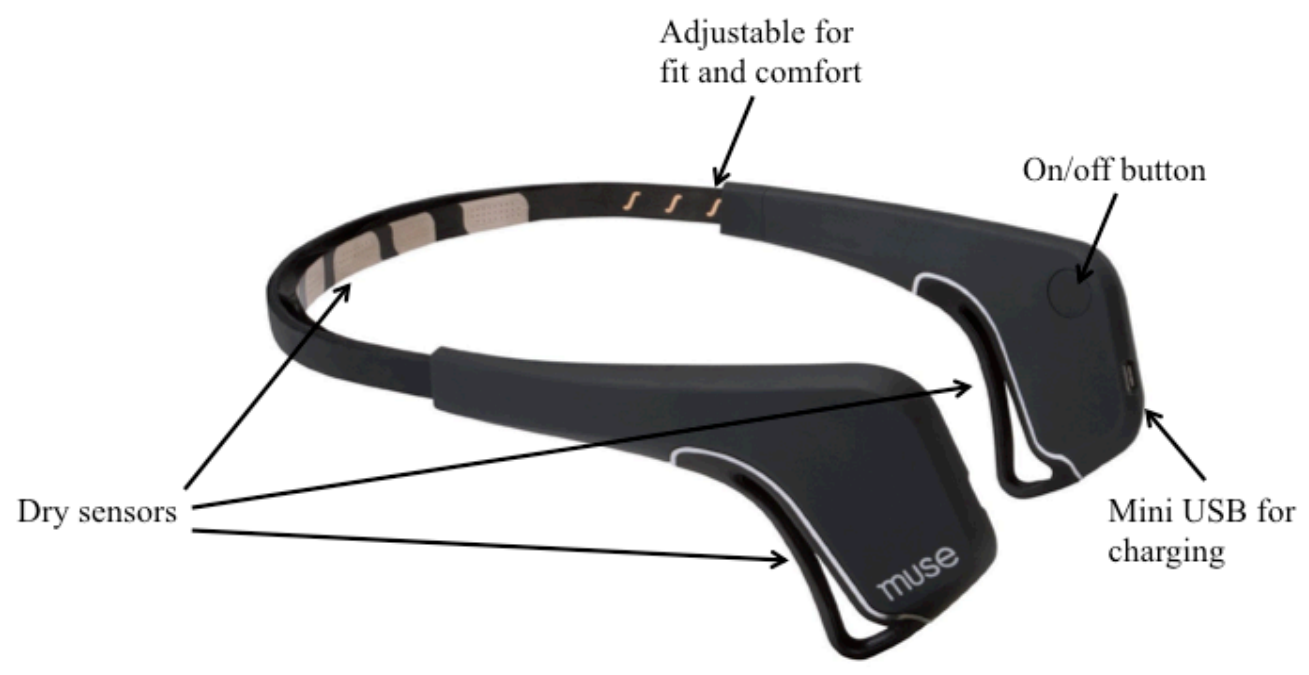

Figure 4. Represented is an image of the Muse Brain-Sensing Headband device.

Muse Calm application. The Muse Calm application (2014) version 1.6.3 for iOS was used to present real-time neurofeedback information from the Muse brainsensing headband. This InteraXon Inc. proprietary software is the primary application created for the Muse brain-sensing headband. In this study, the Calm application was run on an Apple iPad Mini 2. 
A new session was created in the Calm application for each participant. Session Options in the application were arranged so that instructions were on, difficulty was set to easy, the voice was female, and the length of session was 20 minutes (see Figure 5 for an example of the Session Options page).

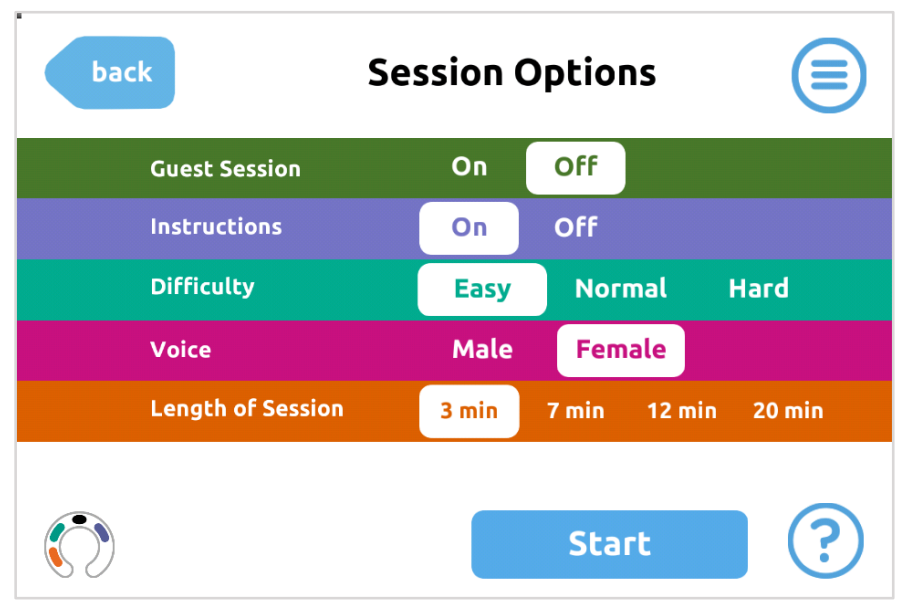

Figure 5. Represented is a screen capture of the Muse Calm application Session Options screen.

At the start of the session, participants underwent calibration, where the Muse device communicated whether it was getting a clear signal from the participant's brainwaves. On the calibration screen (see Figure 6), participants were told to fill the screen with color, with one color representing each of the device sensors. The stronger the brainwave signal to the sensor, the more color appeared on the screen. If one of the sensors was not getting adequate signal, the headband was carefully adjusted on the participant's head until the sensor received a strong brainwave signal. The calibration screen could not be passed until the areas corresponding to all four sensors were filled with solid color. The fifth sensor, which sat in the middle of the participant's forehead, did not require calibration. 


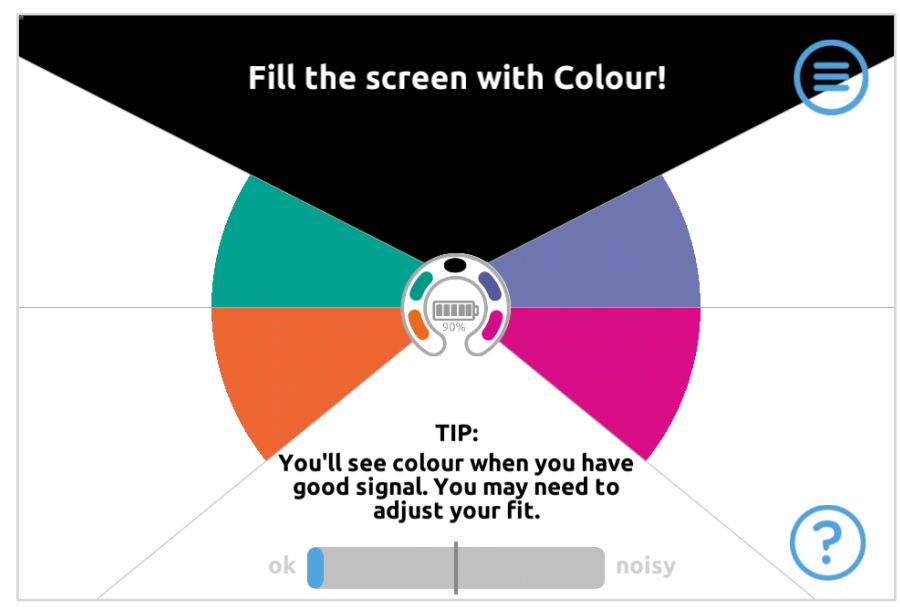

Figure 6. Represented is a screenshot of the Muse Calm application calibration screen.

After initial calibration, the Muse Calm application lead participants through a thought exercise so that the device could establish a baseline of the participant's active brainwaves. First, the application named three different categories of things, one at a time, and the participant should think of as many different items in that category as possible (see Figure 7). Then, the application named the first category and the countdown began (see Figure 8). The calibration lasted for one minute. For example, the application may identify types of fruits, then sports teams, then U.S. cities. The categories presented to each participant were randomly generated by the Muse Calm application. 


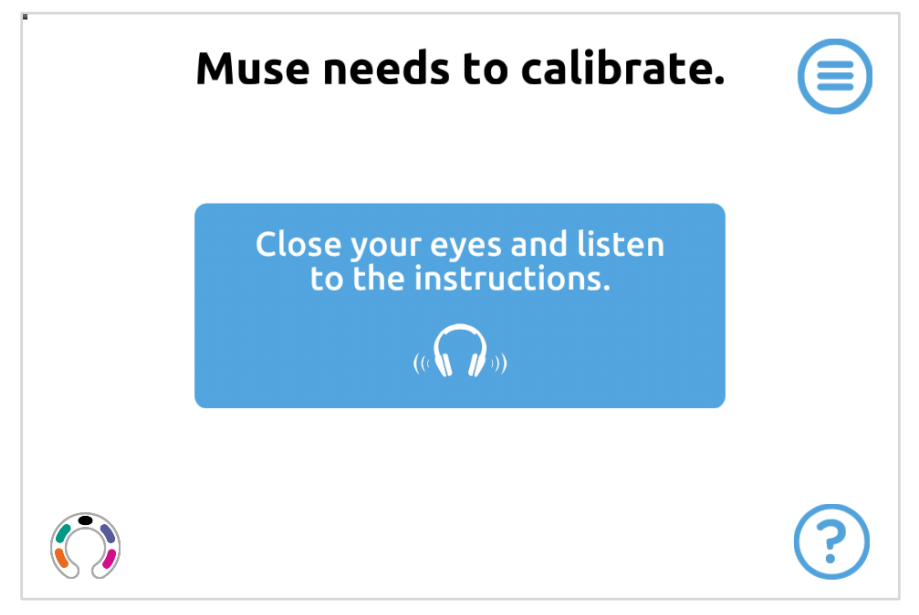

Figure 7. Represented is a screenshot of the Muse Calm application thought calibration instruction screen.

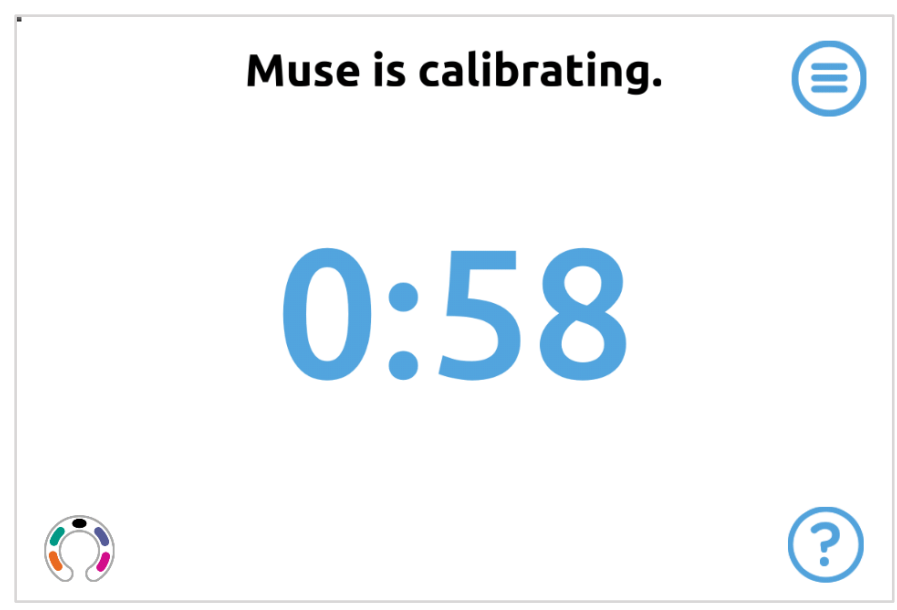

Figure 8. Represented is a screenshot of the Muse Calm application thought calibration countdown screen.

After the thought calibration countdown, the Muse Calm application calibrated the device sensors again to ensure a stable signal (see Figure 6). Finally, a beach scene appeared on the screen and participants were given instructions in mindfulness meditation (see Figure 9). In the instructions, participants were encouraged to close their eyes, sit in an upright and comfortable position, and focus on the breath. After the 
instructions were complete, participants were told to begin and a 20-minute countdown appeared on the screen (see Figure 10 for an example of the countdown screen).

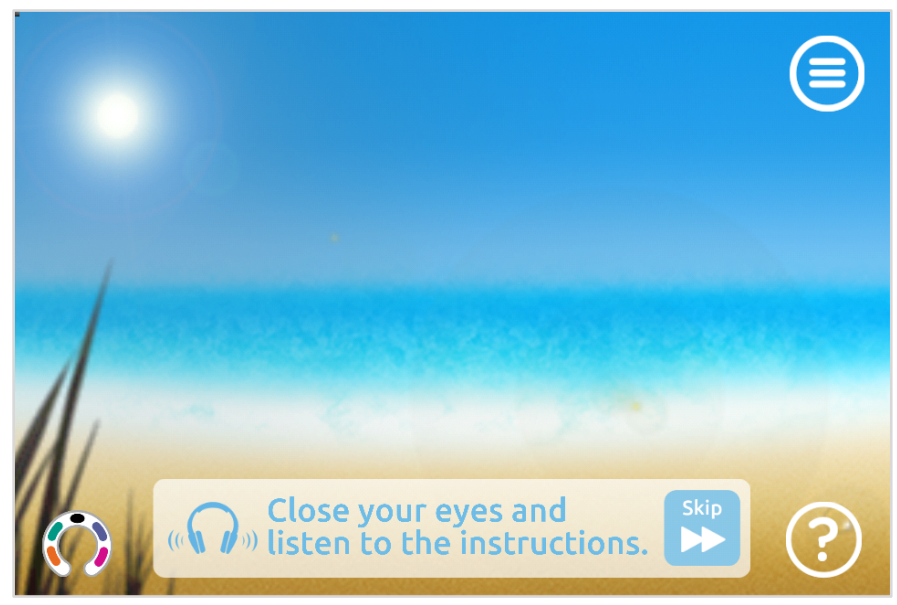

Figure 9. Represented is a screenshot of the Muse Calm application mindfulness meditation instructions screen.

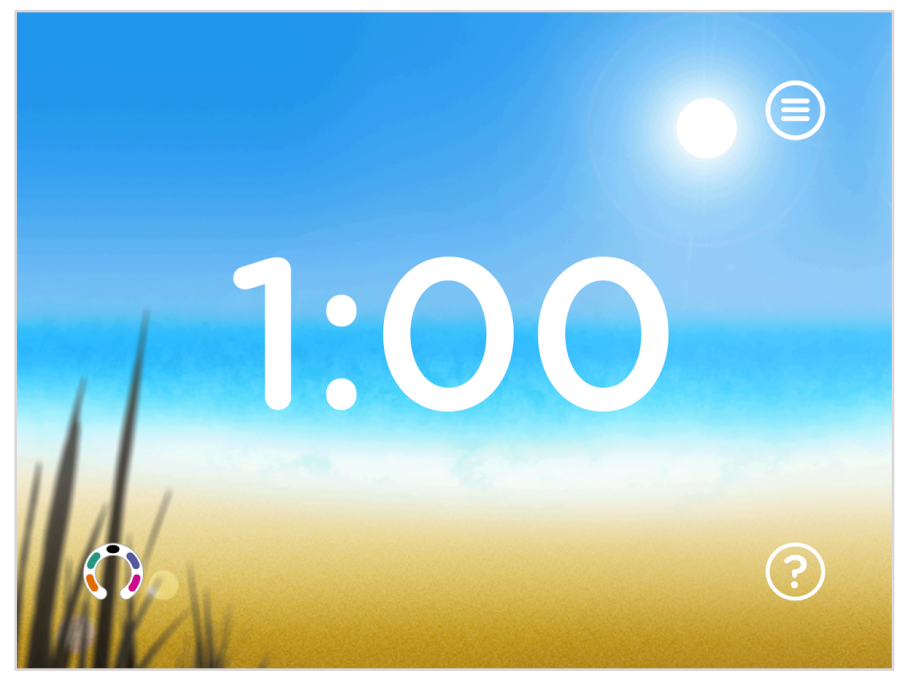

Figure 10. Represented is a screenshot of the Muse Calm application mindfulness meditation countdown screen.

Once the mindfulness meditation session began, participants heard neurofeedback in the form of wind and wave sounds. If a participant's brainwaves were calm, the wind and wave sounds were subtle. If a participant's brainwaves were active, the wind and 
wave sounds were more intense. The beach scene also adjusted as the participant's brainwave activity level changed. Calmer brainwaves were depicted as a clear sky and minimal plant activity (see Figure 11), whereas more active brainwaves were correlated with clouds in the sky and movement in the sea and plants (see Figure 10).

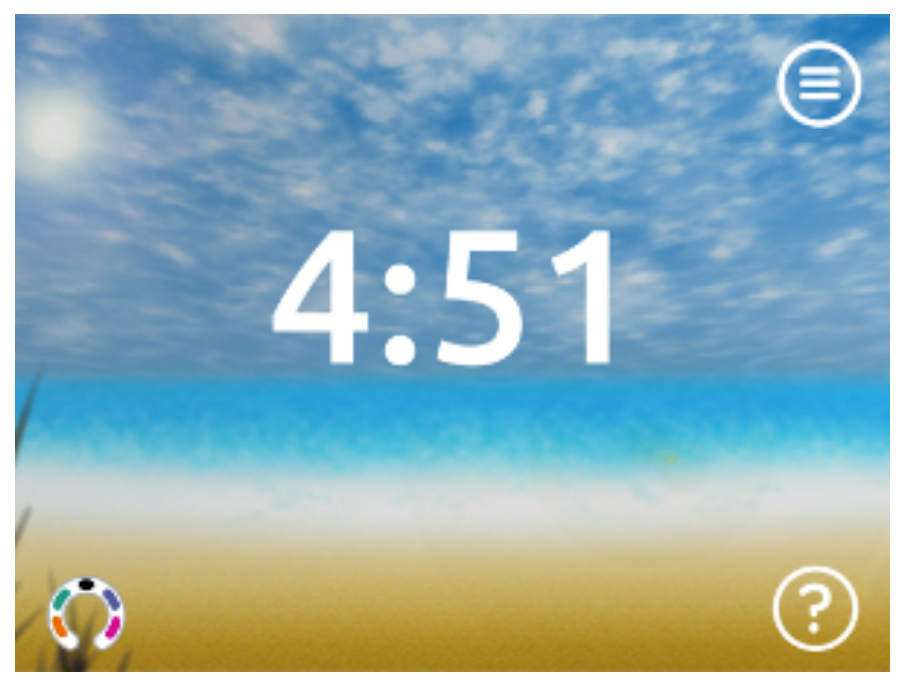

Figure 11. Represented is a screenshot of the Muse Calm application mindfulness meditation countdown screen with clouds and waves, indicating active brainwaves.

Participants in the mindfulness meditation neurofeedback group experienced all aforementioned screens and audio feedback. Participants in the mindfulness meditation group experienced the calibration and mindfulness meditation instruction screens, but then all neurofeedback screens (see Figures 10 and 11) were covered with a cloth and the volume muted so that mindfulness meditation group participants received no audial or visual feedback.

Stopwatch. Stroop task scores were timed to a hundredth of a second by the researcher on an iPhone 4S using the Stopwatch application created by Tim O's Studios version 4.0 for iOS. This stopwatch application was chosen because it prevents the 
phone from sleeping, thereby allowing easy access to Start, Stop, and Reset buttons. After each participant's session the researcher recorded the participant's total time for the Stroop task into a digital spreadsheet and the stopwatch was cleared.

Tally counter. The researcher counted Stroop task errors by a hand tally counter. The researcher recorded the number of errors in a digital spreadsheet.

\section{Procedure}

Participants were screened online using a digital version of the Pre-Experiment Questionnaire (see Appendix B), which included questions on participant physical activity level and mindfulness meditation practices. Participants who met the physical activity and mindfulness meditation requirements were randomly assigned to one of three groups: acute physical exercise, mindfulness meditation neurofeedback, or mindfulness meditation. Prior to the study date, participants were contacted with the location, date, and time of their session and were asked to not engage in structured physical exercise on the day of the session or to consume food, alcohol, or nicotine for three hours prior to the session (ACSM, 2014). Participants were informed that a 20-minute bout of exercise might be required and that they should dress for exercise. Participants were informed that the session would take approximately 45 minutes. All testing took place in an indoor, climate-controlled lab at the university. A summary of the procedures for all groups is presented in Figure 12. 


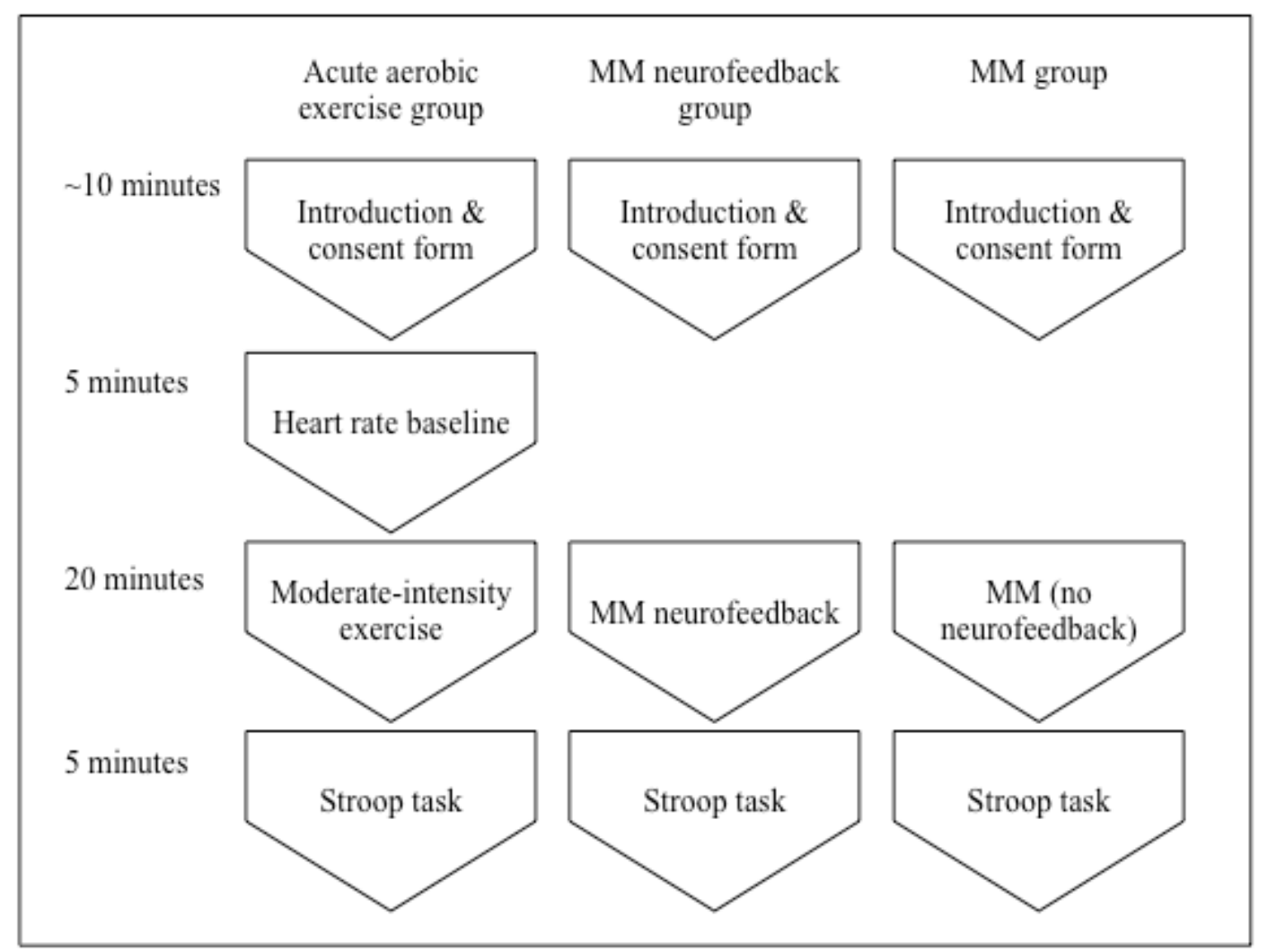

Figure 12. Illustration of a flow chart to indicate the design of the study and procedures for the three intervention groups.

Acute aerobic exercise group. On arrival to the study location, participants reviewed and signed the informed consent form (see Appendix C). Acute aerobic exercise group participants were then asked to review and fill out the PAR-Q form (Appendix A). Participants who answered negatively to all questions in the PAR-Q were permitted to continue with the session. In this study, all participants answered negatively to all questions in the PAR-Q. After the paperwork was completed, the researcher introduced the mechanics of the MIO 0021US-BLK2 Drive+ Strapless Heart Rate Monitor Watch (MIO Global, Vancouver, British Columbia), and the Marcy ME-708 upright stationary bicycle. The MIO heart rate monitor was fitted to the participant's 
wrist and the participant was asked to sit quietly for five minutes to establish a resting heart rate.

After the resting heart rate was recorded, the researcher calculated the participant's HRR. The participant's individual target heart rate was explained to the participant and he or she was asked to keep the heart rate within \pm 5 beats per minute (BPM) of the target heart rate for the 20 minutes following warm-up. Participants were then asked to move to the stationary bicycle where they completed a five-minute warmup. During those five minutes, participants were asked to raise their heart rates to \pm 5 BPM of their target heart rate. After the five-minute warm-up, participants were asked to monitor heart rate and maintain $\pm 5 \mathrm{BPM}$ of their HRR for 20 minutes. At 5, 10, and 15 minute markers, the researcher reminded the participants to check the heart rate monitor to ensure BPM was within range. Following the 20-minute bout of exercise, participants completed 2.5 minutes of cool-down, and then were asked to leave the stationary bicycle and sit in front of a laptop computer. Participants then performed 50 trials of the Stroop task. The researcher recorded the number of errors and total Stroop task time in a digital spreadsheet for each participant. This concluded the procedure for the acute aerobic exercise intervention.

Mindfulness meditation neurofeedback group. Participants reviewed and signed the informed consent form (see Appendix C) and were informed that they would be experiencing mindfulness meditation neurofeedback during the session. The researcher introduced the Muse brain-sensing headband by InteraXon, Inc. The Muse device was fit comfortably on the participant's head. The researcher then started a new 
session in the Calm application. The Calm application led the participant through device calibration and gave mindfulness meditation instructions. Following the mindfulness mediation instructions, the participant completed 20 minutes of mindfulness meditation neurofeedback. Following the mindfulness meditation neurofeedback session, the researcher removed the Muse headband and the participant moved to the laptop computer for 50 trials of the Stroop task. The researcher recorded the number of errors and total Stroop task time in a digital spreadsheet for each participant. This concluded the procedure for the mindfulness meditation neurofeedback intervention.

Mindfulness meditation group. Participants reviewed and signed the informed consent form (see Appendix C). Then, the researcher introduced the Muse brain-sensing headband by InteraXon, Inc. and fit the device comfortably on the participant's head. The researcher then started a new session in the Calm application. The Calm application led the participant through device calibration and gave mindfulness meditation instructions. Following the mindfulness mediation instructions, the researcher turned off the volume on the iPad and covered the screen with an opaque cloth so that the participant was left without feedback for 20 minutes of mindfulness meditation. Following the mindfulness meditation session, the researcher removed the Muse headband and the participant moved to the laptop computer for 50 trials of the Stroop task. The researcher recorded the number of errors and total Stroop task time in a digital spreadsheet for each participant. This concluded the mindfulness meditation group's participation. 


\section{Chapter 4}

\section{Results}

\section{Participants}

Of those recruited, nine individuals were excluded from this study because they did not meet the minimum exercise requirements, i.e., 150 minutes or more moderate intensity exercise per week or 75 minutes or more of high intensity exercise per week (ACSM, 2014). Two additional individuals were excluded due to colorblindness. One additional individual was excluded due to regular meditation practice. In total, this left 42 participants who took part in the study. All 42 participants underwent one intervention, either acute physical exercise, mindfulness meditation, or mindfulness meditation neurofeedback, and Stroop time and Stroop number of errors were collected from all participants. There were 14 participants per intervention.

Participant demographic data are contained in Table 1. The sample included male (41\%) and female (59\%) participants ages $18-45$ years. An analysis of the frequency distribution of participant demographic data indicated that neither gender nor age group appears to have had an effect on the results of this study. 
Table 1

Participant Demographics by Intervention Group

\begin{tabular}{ccccc}
\hline Variable & $\begin{array}{c}\text { Physical exercise } \\
\text { Percentage (n) }\end{array}$ & $\begin{array}{c}\text { Mindfulness meditation } \\
\text { Percentage (n) }\end{array}$ & $\begin{array}{c}\text { MM neurofeedback } \\
\text { Percentage (n) }\end{array}$ & $\begin{array}{c}\text { Total } \\
\text { Percentage (n) }\end{array}$ \\
\hline $\begin{array}{c}\text { Age (years) } \\
18-25\end{array}$ & $93(13)$ & $71(10)$ & $79(11)$ & $81(34)$ \\
$26-35$ & $0(0)$ & $21(3)$ & $14(2)$ & $12(5)$ \\
$36-45$ & $7(1)$ & $7(1)$ & $7(1)$ & $7(3)$ \\
Gender & & & & \\
Female & $64(9)$ & $57(8)$ & $57(8)$ & $60(25)$ \\
Male & $36(5)$ & $43(6)$ & $43(6)$ & $40(17)$ \\
\hline
\end{tabular}

Note: Values may not sum to $100 \%$ due to rounding. Mindfulness meditation is abbreviated to "MM."

On the Pre-experiment Questionnaire (Appendix B), all included participants indicated that they engage in 150 minutes or more moderate intensity exercise per week or 75 minutes or more of high intensity exercise per week (ACSM, 2014). In the Preexperiment Questionnaire, moderate intensity exercise was described as brisk walking or cycling on level ground. High intensity exercise was described as running or swimming laps. On the questionnaire, participants may have chosen that they engaged in moderate intensity exercise, high intensity exercise, or both. Of the total, 24 participants indicated that they engage in weekly moderate intensity exercise, and 28 participants indicated that they engage in weekly high intensity exercise. There were 10 participants who indicated that they engage in both moderate and high intensity exercise on a weekly basis. 
Data from all participants who indicated that they engage in moderate intensity exercise $(n=24)$ are found in Table 2. Data from all participants who indicated that they engage in high intensity exercise $(n=28)$ are found in Table 3 . Tables 2 and 3 contain participant frequency data for the number of days per week spent exercising and the duration spent exercising in a single session.

The majority of moderate intensity exercisers indicated that they engage in exercise 5 days per week (46\%) for 30-45 minutes (29\%) or 45-60 minutes (29\%) sessions. The majority of high intensity exercisers indicated that they engage in exercise 4 days per week (43\%) for 45-60 minute sessions (36\%).

Participant responses to whether they engage in moderate or high intensity exercise were not taken into account in assigning participants to intervention groups. The instrument was only used to determine inclusion or exclusion in the study based on minimum exercise requirements. For participants who indicated that they engage in moderate intensity exercise, $33 \%$ were assigned to the physical exercise group, $29 \%$ to the mindfulness meditation group, and $38 \%$ to the mindfulness meditation neurofeedback group. For participants who indicated that they engage in high intensity exercise, $32 \%$ were assigned to the physical exercise group, $32 \%$ to the mindfulness meditation group, and $36 \%$ to the mindfulness meditation neurofeedback group. 
Table 2

Participant Self-Reported Frequency and Duration of Moderate Intensity Aerobic Exercise by Intervention Group

\begin{tabular}{|c|c|c|c|c|}
\hline Variable & $\frac{\text { Physical exercise }}{\text { Percentage (n) }}$ & $\frac{\text { Mindfulness meditation }}{\text { Percentage (n) }}$ & $\frac{\text { MM neurofeedback }}{\text { Percentage (n) }}$ & $\begin{array}{c}\text { Total } \\
\text { Percentage (n) }\end{array}$ \\
\hline \multicolumn{5}{|c|}{ Frequency of exercise (days per week) } \\
\hline 3 & $7(1)$ & $0(0)$ & $7(1)$ & $8(2)$ \\
\hline 4 & $7(1)$ & $29(4)$ & $29(4)$ & $38(9)$ \\
\hline 5 & $29(4)$ & $21(3)$ & $29(4)$ & $46(11)$ \\
\hline 6 & $14(2)$ & $0(0)$ & $0(0)$ & $8(2)$ \\
\hline 7 & $0(0)$ & $0(0)$ & $0(0)$ & $0(0)$ \\
\hline \multicolumn{5}{|c|}{ Duration of exercise (minutes per session) } \\
\hline $20-30$ & $7(1)$ & $7(1)$ & $14(2)$ & $17(4)$ \\
\hline $30-45$ & $0(0)$ & $21(3)$ & $29(4)$ & $29(7)$ \\
\hline $45-60$ & $21(3)$ & $21(3)$ & $7(1)$ & $29(7)$ \\
\hline $60+$ & $29(4)$ & $0(0)$ & $14(2)$ & $25(6)$ \\
\hline
\end{tabular}

Note. $n=24$ for self-reported weekly moderate intensity aerobic exercise. Participants may have chosen both moderate intensity and high intensity in the pre-experiment questionnaire. Values may not sum to $100 \%$ due to rounding. Mindfulness meditation is abbreviated to "MM." 
Table 3

Participant Self-Reported Frequency and Duration of High Intensity Aerobic Exercise by Intervention Group

\begin{tabular}{|c|c|c|c|c|}
\hline & Physical exercise & Mindfulness meditation & MM neurofeedback & Total \\
\hline Variable & Percentage (n) & Percentage (n) & Percentage (n) & Percentage $(n)$ \\
\hline \multicolumn{5}{|c|}{ Frequency of exercise (days per week) } \\
\hline 3 & $7(1)$ & $21(3)$ & $14(2)$ & $21(6)$ \\
\hline 4 & $14(2)$ & $36(5)$ & $36(5)$ & $43(12)$ \\
\hline 5 & $7(1)$ & $7(1)$ & $14(2)$ & $14(4)$ \\
\hline 6 & $29(4)$ & $0(0)$ & $0(0)$ & $14(4)$ \\
\hline 7 & $7(1)$ & $0(0)$ & $7(1)$ & $7(2)$ \\
\hline \multicolumn{5}{|c|}{ Duration of exercise (minutes per session) } \\
\hline $20-30$ & $0(0)$ & $0(0)$ & $0(0)$ & $0(0)$ \\
\hline $30-45$ & $7(1)$ & $21(3)$ & $36(5)$ & $32(9)$ \\
\hline $45-60$ & $29(4)$ & $29(4)$ & $14(2)$ & $36(10)$ \\
\hline $60+$ & $29(4)$ & $14(2)$ & $21(3)$ & $32(9)$ \\
\hline
\end{tabular}

Note. $n=28$ for self-reported weekly high intensity aerobic exercise. Participants may have chosen both moderate intensity and high intensity in the pre-experiment questionnaire. Values may not sum to $100 \%$ due to rounding. Mindfulness meditation is abbreviated to "MM."

\section{Effect of study interventions on Stroop task time}

All data were analyzed using IBM SPSS Statistics version 22. The total time that it took each participant to complete all 50 trials of the Stroop task contributed to a participant's Stroop task response time. Table 4 depicts the mean and standard deviation for Stroop task response time by intervention group. 
Table 4

Descriptive Statistics for Stroop Task Time by Intervention Group

\begin{tabular}{lcc}
\hline Intervention group & \multicolumn{2}{c}{ Stroop time (in seconds) } \\
\hline & $M$ & $S D$ \\
\cline { 2 - 3 } Acute Physical Exercise & 59.07 & 7.41 \\
Mindfulness meditation & 63.07 & 7.78 \\
Mindfulness meditation Neurofeedback & 60.14 & 11.03 \\
\hline
\end{tabular}

A one-way ANOVA was conducted to investigate any differences in Stroop task response time between the three intervention groups, acute physical exercise, mindfulness meditation, and mindfulness meditation neurofeedback. There was homogeneity of variances, as assessed by Levene's test for equality of variances $(p=.165)$. No statistically significant effects were found for intervention group on Stroop time $F(2,29)$ $=.759, p=.475$.

\section{Effect of study interventions on Stroop task number of errors}

The number of errors each participant made while completing the 50-trials of the Stroop task were recorded by the researcher. During the Stroop task, participants made between zero and two errors. Table 5 depicts the mean and standard deviation for the Stroop task number of errors for each intervention group. The means for the three intervention groups vary considerably; however the standard deviations are more similar, especially for the acute physical exercise and mindfulness meditation neurofeedback groups. This indicates that spread of data points is similar across those intervention 
groups. In the case of Stroop task number of errors, a small mean and small standard deviation are desirable, as that indicates fewer errors made by the intervention group.

Table 5

Descriptive Statistics for Stroop Task Number of Errors by Intervention Group

\begin{tabular}{lcc}
\hline Intervention group & \multicolumn{2}{c}{ Stroop Number of Errors } \\
\hline & $M$ & $S D$ \\
\cline { 2 - 3 } Acute physical exercise & 0.93 & 0.62 \\
Mindfulness meditation & 0.50 & 0.76 \\
Mindfulness meditation neurofeedback & 0.29 & 0.61 \\
\hline
\end{tabular}

A one-way ANOVA was conducted to investigate any differences in Stroop task number of errors between the three intervention groups, acute physical exercise, mindfulness meditation, and mindfulness meditation neurofeedback. A Levene's test for equality of variances $(p=.256)$ supported the assumption of homogeneity of variance. Stroop number of errors was found to be statistically significant between intervention groups $F(2,39)=3.384, p=.044$. A post hoc Tukey's HSD test indicated that the mindfulness meditation neurofeedback intervention group had significantly fewer errors than did the acute exercise intervention group $(p=.038)$. Statically significant results were not observed for the other pairwise comparisons.

A frequency distribution of the Stroop task number of errors broken out by intervention group can be found in Figure 13. The mindfulness meditation neurofeedback group contained the most of participants who made zero mistakes (11) 
followed by the mindfulness meditation group (9) and then the physical exercise group (3). The majority of participants in the physical exercise group (9) made one error. Three participants in the mindfulness meditation group made one error and two participants in the mindfulness meditation neurofeedback group made one error. The physical exercise and mindfulness meditation groups contained equal number of participants who made two errors (2), while in the mindfulness meditation neurofeedback group only one participant made two errors. A visual analysis of these frequencies confirms the hypothesis test results: The number of participants who made zero errors is much greater in the mindfulness meditation neurofeedback group than the acute physical exercise group. From these data, it appears that mindfulness meditation neurofeedback aided participants in making fewer errors on the Stroop task when compared to the physical exercise condition. The error frequency pattern is more similar for the mindfulness meditation neurofeedback and mindfulness meditation groups. This may suggest that mindfulness meditation also aided participants to make fewer errors on the Stroop task, but no to the extent that adding in neurofeedback did. 


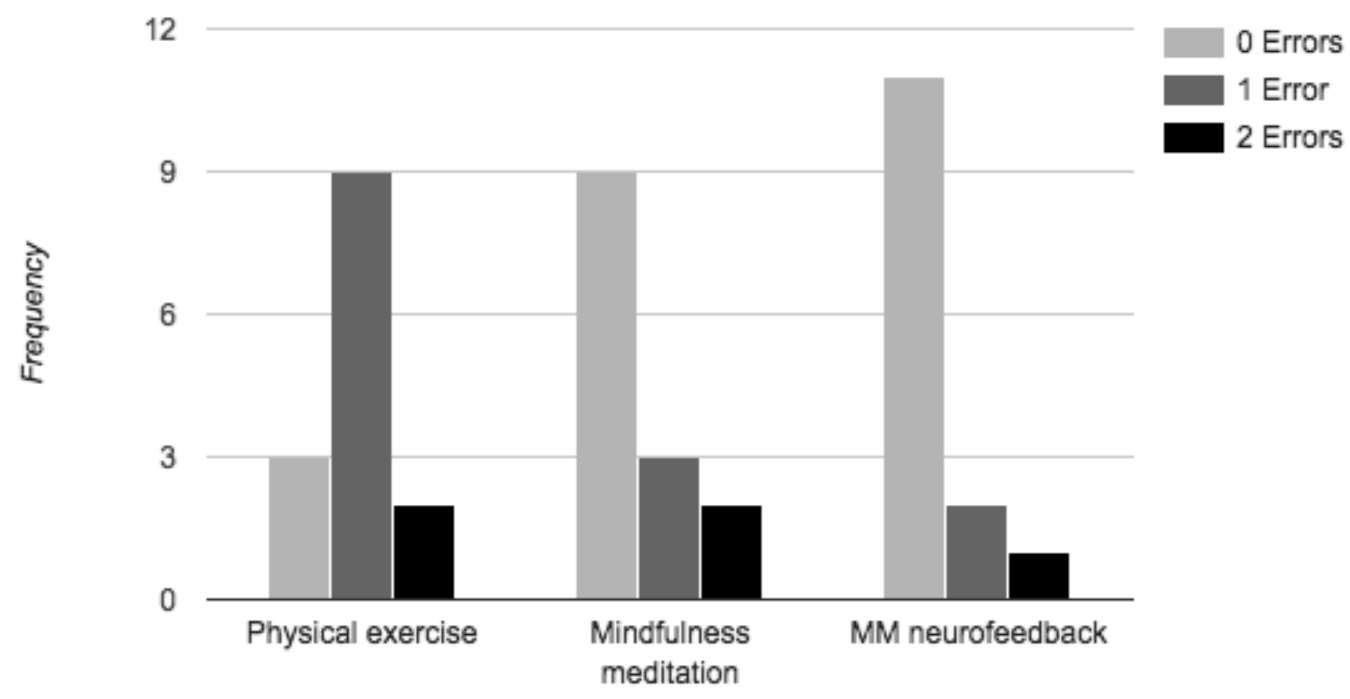

Figure 13. Frequency distribution representing the Stroop task number of errors by intervention group.

Effect size was measured using Cohen's $d$. Results are presented in Table 6. Cohen's $d$ indicates a large mean effect size for the physical exercise - mindfulness meditation neurofeedback pairwise comparison.

Table 6

Pairwise Comparisons for Stroop Task Number of Errors

\begin{tabular}{lcc}
\hline Intervention group comparison & $p$ & $d$ \\
\hline Physical exercise - Mindfulness meditation & .167 & 0.60 \\
Physical exercise - Mindfulness meditation neurofeedback & .020 & 1.08 \\
Mindfulness meditation neurofeedback - Mindfulness meditation & 1.0 & 0.32 \\
\hline
\end{tabular}




\section{Summary of Results}

The present study tested the effects of three interventions, acute physical exercise, mindfulness meditation, and mindfulness meditation neurofeedback, on Stroop task time and number of errors. Forty-two individuals participated in the study, 14 participants in each of the three intervention groups. The sample included male (41\%) and female (59\%) participants ages $18-45$ years.

The total time that it took each participant to complete all 50 trials of the Stroop task contributed to a participant's Stroop task time. A one-way ANOVA was conducted to investigate any differences in Stroop task time between the three intervention groups. No statistically significant effects were found for intervention group on Stroop time $F(2,29)=.759, p=.475$

The number of errors each participant made while completing the 50-trials of the Stroop task (0-2 errors) were recorded by the researcher and contributed to the participant's Stroop task number of errors. A one-way ANOVA was conducted to investigate any differences in Stroop task number of errors between the three intervention groups. Stroop number of errors were found to be statistically significant between groups $F(2,39)=3.384, p=.044$. Pairwise comparisons were performed using Tukey's HSD. This post hoc analysis revealed statistically significant differences in Stroop task number of errors between the acute physical exercise and mindfulness meditation neurofeedback intervention groups $(p=.038)$, but not between any other intervention group combinations. Cohen's $d$ indicates a large mean effect size for the physical exercise mindfulness meditation neurofeedback pairwise comparison $d=1.08$. 


\section{Chapter 5}

\section{Discussion}

The purpose of this study was to test the between subject effects of an acute bout of exercise, a single session of mindfulness meditation neurofeedback, and a single session of mindfulness meditation on cognitive performance in a sample of healthy adults. Test of mindfulness meditation neurofeedback as an intervention is novel. Thus, this is a preliminary investigation into the ideas studied here and not a confirmatory investigation.

Results of this study indicate that there is a significant difference in postintervention Stroop task errors between the acute physical exercise and mindfulness meditation neurofeedback groups. Participants made fewer errors after undergoing the mindfulness meditation neurofeedback intervention than they did following acute physical exercise. Previous research that investigated cognitive performance following acute physical activity found small $(d=\sim .20)$ but significant effects for that intervention (Etnier et al., 1997). In this study, effect size for the mean difference between the acute physical exercise and mindfulness meditation neurofeedback groups was found to be large $(d=1.08)$. The inclusion of mindfulness meditation neurofeedback as an intervention is one reason why this effect size is larger than other studies investigating effects of acute physical activity. The finding that Stroop task number of errors was fewer for the mindfulness meditation neurofeedback group than the acute physical exercise group suggests that mindfulness meditation neurofeedback may be an effective method for certain individuals to boost their executive function. 


\section{Limitations and Future Directions}

Stroop task time data collection. The method used to measure Stroop task time may have contaminated Stroop task time. In this study, the researcher manually tracked Stroop task time using a mobile phone stopwatch that counted to hundredths of seconds. As such, the researcher's reaction time was counted in addition to participants' response times. The researcher's reaction time may have improved over time, having a faster reaction time for participants timed later in the study or during latter trials boredom might have played a role on the time variable. The researcher's reaction time may be considered random error in this study, however, because it was spread equally across groups, i.e., assignment of participants to intervention groups was random, thus creating a systematic bias.

Future studies that wish to calculate Stroop task time may gain more accurate results by using a computerized version of the Stroop task. Computerized versions of the Stroop task that accept voice input, such as was used in research by Barella et al. (2010), would be ideal because they would be more accurate than manual timing by the researcher while allowing for natural verbal response from participants. Other computerized versions require participants to select a button on a keyboard to indicate the color of their answer. This method would also be more accurate than manual timing; however, it could add cognitive load represented in the corresponding pair of keyboard button with color in the Stroop task. Researchers wanting to use a computerized Stroop task without voice input may consider controlling for participant familiarity with touchtyping on a QWERTY keyboard. 
Stroop number of errors contingency analysis. A chi-square contingency table analysis was conducted with the Stroop number of errors data to better understand whether intervention group and Stroop number of errors were associated. While the Stroop error data are numerical, they are not continuous because it is not possible to have a fraction of an error nor are their ranges greater than two errors. As such, the chi-square analysis requirement of categorical variables was met. Unfortunately, however, the analysis resulted in expected cell frequencies that were not greater than five (see Table 7), indicating that the data may be spurious. However, anecdotal insights may still be gathered from these data.

Table 7

Chi-Square Contingency Table Expected and Actual Stroop Number of Errors

\begin{tabular}{ccccc}
\hline $\begin{array}{c}\text { Stroop } \\
\text { errors }\end{array}$ & $\begin{array}{c}\text { Expected } \\
\text { count }\end{array}$ & $\begin{array}{c}\text { Physical exercise } \\
\text { Actual count }\end{array}$ & $\begin{array}{c}\text { Mindfulness meditation } \\
\text { Actual count }\end{array}$ & $\begin{array}{c}\text { MM neurofeedback } \\
\text { Actual count }\end{array}$ \\
0 errors & 7.7 & 3 & 9 & 11 \\
1 error & 4.7 & 9 & 3 & 2 \\
2 errors & 1.7 & 2 & 2 & 1 \\
\hline
\end{tabular}

The chi-square contingency table expected counts for Stroop number of errors was 7.7 participants with zero errors, 4.7 participants with one error, and 1.7 participants with 2 errors. The acute physical exercise group had nearly double the amount of expected participants with one error and less than half of the expected number of participants with zero errors. Both the mindfulness meditation and the mindfulness 
meditation neurofeedback groups contained more than the expected number of participants who made zero errors and fewer than the expected number of participants with one error. From these descriptive data, it appears that the acute physical exercise group made more error than the mindfulness meditation and mindfulness meditation neurofeedback groups.

Single session, between subjects study design. The majority of research on mindfulness meditation and neurofeedback includes multiple sessions with the interventions. Similarly, while this study focused on acute physical exercise, chronic exercise programs that extend over the course of weeks or months have been shown to benefit cognitive performance (Chang \& Etnier, 2014). Limited resources restricted the present study to a single session with mindfulness meditation neurofeedback or mindfulness meditation; however future research may consider a conducting a repeated measures study that may more accurately test the effects of these interventions over time.

Future research may also consider breaking down Stroop trials into 10 or 20-trial chunks for comparison analysis over the course of a 50 or 100-trial repeated measures test to understand if there are any cognitive performance effects during the duration of the cognitive task. Similarly, Stroop time and number of errors may be tested across time, perhaps immediately following intervention, minutes later, and an hour later, to test any longer-term latency effects of the intervention.

A between subjects design was chosen for this study to avoid carryover effects from the three interventions. However, the addition of a within subjects design may be 
considered in which participants would undergo all three interventions, acute physical exercise, mindfulness meditation, and mindfulness meditation neurofeedback. A within subjects design would reduce the effects of individual differences and, given enough time between interventions, carryover effects may be reduced.

Expectancy effects of the Muse device. Some participants may have experienced expectancy effects simply by wearing the Muse headband, which would pose a threat to the internal validity of the study (Campbell \& Stanley, 2963; Cook \& Campbell, 1979). To mitigate the threat of expectancy effects, the mindfulness meditation intervention group was included so that a second group of participants would wear the device, but undergo the neurofeedback intervention, thereby providing a point of comparison between the mindfulness meditation and mindfulness meditation neurofeedback groups. Future studies may consider using this method to control expectancy effects or add a control group that wears the Muse headband but receives no instructions or feedback whatsoever.

Participant sample. The majority of the participants in the study (78\%) were between the ages of 18 and 25 years and $59 \%$ of the participants were female. These two limitations may reduce the generalizability of the results to wider populations. For safety reasons, participants were restricted to individuals who engage in at least the minimum amount of aerobic physical exercise recommended by the American College of Sports Medicine (ACMS, 2014). These chronic exercisers may have different results from people with varying levels of physical fitness. Future research investigating acute physical exercise, mindfulness meditation neurofeedback, and mindfulness meditation 
should consider including individuals with varying levels of physical fitness, as past research indicates that fitness level may impact the extent to which exercises improves cognitive performance (Chang et al., 2014).

Stroop task as a measure of executive function. Although Etnier and Chang (2009) reported that the Stroop task is one of the most frequently used tests of executive function, the present study observed little variation in Stroop errors across all intervention groups. This finding along with results from similar studies (Alves et al., 2014; Sibley et al., 2006) suggests that the Stroop test may not be a sensitive measure of error. Future studies may consider other tests of executive function, such as the Tower of London task.

Acute physical exercise intensity. The acute aerobic exercise intervention in this study included only moderate-intensity exercise. Moderate-intensity exercise was chosen because previous research has shown that moderate-intensity produced the greatest effects on cognitive performance when compared to low or high-intensity exercise (Chang et al., 2012). Nevertheless, moderate-intensity exercise may have limited the differences observed in participant Stroop task times and number of errors. Including participants with more sedentary lifestyles may help to observe greater or lesser gains in executive performance following intervention. Future research may investigate different levels of exercise intensity to better understand its effects on Stroop time and number of errors.

Lack of interrater reliability. Interrrater reliability was not established for the present study due to time and resource constraints. However, the addition of an 
independent observer to quantify participant errors on the Stroop task would increase data reliability. Future studies may consider building two or more observers into the study design.

Heart rate as a measure. In the present study, heart rate was measured in the acute physical exercise group only as a way to ensure that the participant was experiencing moderate intensity exercise. Future studies may consider including heart rate as a measure of physical activity or calmness across intervention groups. Interesting observations could be made regarding heart rate following mindfulness meditation and mindfulness meditation neurofeedback compared to physical exercise and how heart rate may influence cognitive task performance.

Additional data from the Muse device. At the end of each session, the Muse device provides the individual with a graph of his or her brain activity during the session (See Figure 13) and a percentage of time spent with active, neutral, and calm brainwaves (See Figure 14). Three independent samples t-tests were conducted to determine if there were differences between the mindfulness meditation group and the mindfulness meditation neurofeedback group and the percentage of time their brainwaves were calm, active, and neutral during the 20 -minute session. No statistically significant differences were found between the intervention groups for percentage of time spent in calm, active, or neutral brainwaves. For percentage of time spent with calm brainwaves, $t(26)=0.694$, $p=.494$. For percentage of time spent with active brainwaves, $t(26)=-.236, p=.815$. For percentage of time spent with neutral brainwaves, $t(26)=-0.606, p=.550$. One possible reason that statistically significant differences was not observed is that this study 
used a single-session design and participants may have been nervous or unsure about using the device. A longitudinal study may observe brainwave activity trends over time for neurofeedback and non-neurofeedback groups. Future research may consider making further use of the graphs and percentages that the Muse device generates to investigate the efficacy of the Muse device as an intervention.

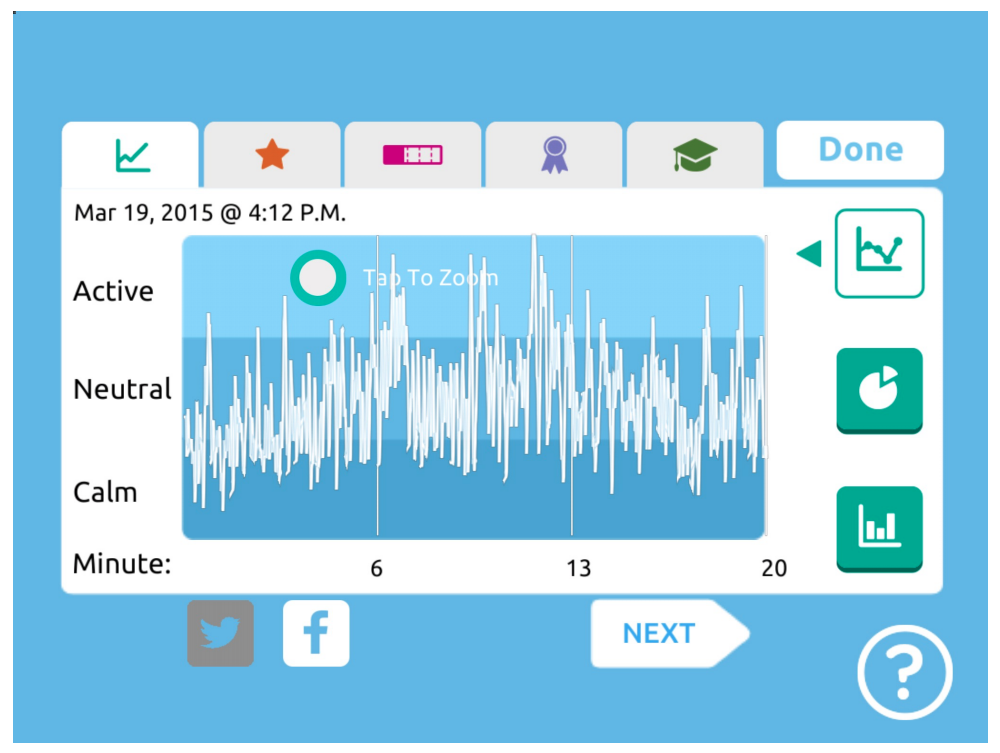

Figure 13. Represented is an example of the post-session graphical output from the Muse device. 


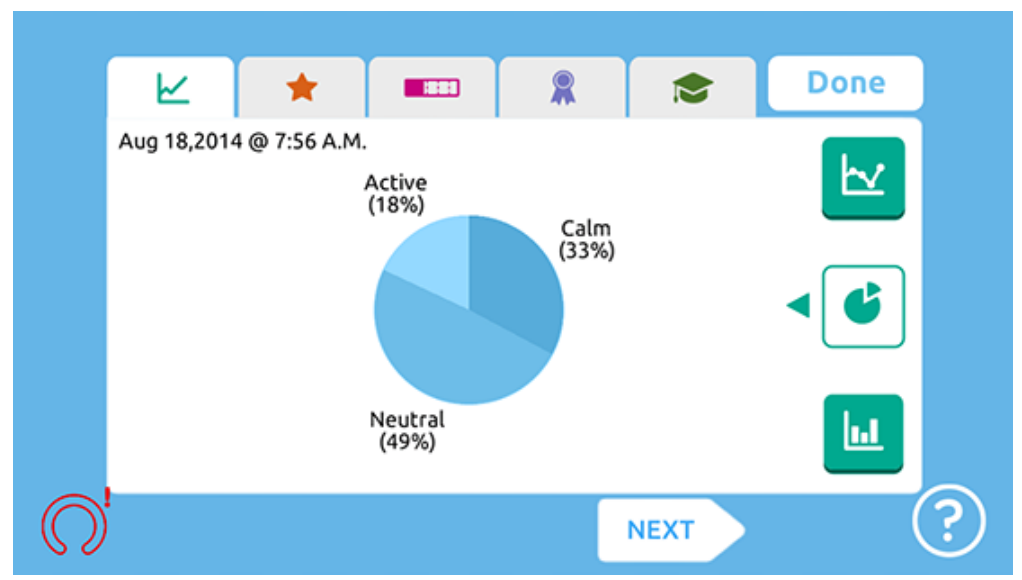

Figure 14. Represented is an example of the post-session percentage output from the Muse device.

Simultaneous interventions. Recent research by Stein, Crowley, Dunnam, and Anderson-Hanley (2014) demonstrated that simultaneous pairing of physical exercise and mental challenge results in a significant improvement in executive function. This research is highly relevant to modern ergonomic exercise workstations, which combine an activity such as walking on a treadmill or pedaling on an ergometer cycle, with a computer workstation. Future research could build from this idea to investigate the effects of physical activity combined with mindfulness meditation or mindfulness meditation neurofeedback on cognitive performance.

\section{Practical implications}

Research has shown that acute physical activity, mindfulness meditation, and neurofeedback have positive effects on executive function. However, each of these interventions has a downside, especially for individuals who have limited time or mobility. Physical exercise requires a certain amount of mobility and people with mobility impairments, whether they are physical or environmental, may have difficulty 
raising their heart rates to a level that constitutes moderate intensity exercise. For people with limited time, learning to meditate without guidance may feel daunting and frustrating. Access to traditional neurofeedback technology is also a limitation for the general population, as EEG and similar technologies are costly and difficult or impossible to use without a trained technician. The new intervention that teaches mindfulness meditation through neurofeedback may have positive effects on executive function for individuals for which the aforementioned interventions are not feasible.

\section{Conclusion}

This study investigated the effects of three interventions on executive function: acute physical exercise, mindfulness meditation, and mindfulness meditation neurofeedback. A statistically significant one-way ANOVA revealed that participants made fewer errors after undergoing the mindfulness meditation neurofeedback intervention than they did following acute physical exercise. These findings demonstrate that mindfulness meditation neurofeedback may boost aspects of executive function for some individuals.

This study is unique in its investigation of the effects of acute physical exercise, mindfulness meditation, and mindfulness meditation neurofeedback on cognitive performance. Two studies have compared the effects of mindfulness meditation and neurofeedback (Lai et al., 2014; Chow, 2014); however thus far no studies have examined mindfulness meditation and neurofeedback as a single intervention, nor have any studies so far tested these three interventions together. While the Muse device by InteraXon, Inc. is currently the only technology that delivers mindfulness meditation 
neurofeedback, iterations on the Muse device and the creation of other similar devices warrant scientific investigation into the capabilities and claims of the devices. In addition to testing the initial effects of cognitive performance-enhancing devices, continued evaluation of the Muse and other devices will require new evaluation methodologies. Examining the effects of mindfulness meditation neurofeedback alongside two other established interventions may constitute a framework that could be used to track the effects and progression of mindfulness meditation neurofeedback devices as they evolve in the market. 


\section{References}

Alves, C.R.R., Tessaro, V.H., Teixeira, L.A.C., Murakava, K., Roschel, H., Gualano, B., \& Takito, M.Y. (2014). Influence of acute high-intensity aerobic interval exercise bout on selective attention and short-term memory tasks. Perceptual \& Motor Skills: Learning \& Memory, 118(1), 63-72.

American College of Sports Medicine (2014). ACSM's guidelines for exercise testing and prescription $\left(9^{\text {th }} \mathrm{ed}\right)$. New York: Lippincott Williams and Wilkins.

Barella, L. A., Etnier, J. L., \& Chang, Y.-K. (2010). The immediate and delayed effects of an acute bout of exercise on cognitive performance of healthy older adults. Journal of Aging and Physical Activity, 18(1), 87-98.

Barenberg, J., Berse, T., \& Dutke, S. (2015). Ergometer cycling enhances executive control in task switching. Journal of Cognitive Psychology, 27(6), 692-703. http://doi.org/10.1080/20445911.2015.1024256

Bink, M., van Nieuwenhuizen, C., Popma, A., Bongers, I. L., \& van Boxtel, G. J. M. (2014). Neurocognitive effects of neurofeedback in adolescents with ADHD: a randomized controlled trial. The Journal of Clinical Psychiatry, 75(5), 535-542. doi:10.4088/JCP.13m08590

Black, J. E., Isaacs, K. R., Anderson, B. J., Alcantara, A. A., \& Greenough, W. T. (1990). Learning causes synaptogenesis, whereas motor activity causes angiogenesis, in cerebellar cortex of adult rats. Proceedings of the National Academy of Sciences of the United States of America, 87(14), 5568-5572.

Boucard, G. K., Albinet, C. T., Bugaiska, A., Bouquet, C. A., Clarys, D., \& Audiffren, M. (2012). Impact of Physical Activity on Executive Functions in Aging: A Selective Effect on Inhibition Among Old Adults. Journal of Sport \& Exercise Psychology, 34(6), 808-827.

Brandmeyer, T., \& Delorme, A. (2013). Meditation and neurofeedback. Frontiers in Psychology, 4. doi:10.3389/fpsyg.2013.00688

Brisswalter, J., Collardeau, M., \& René, A. (2002). Effects of acute physical exercise characteristics on cognitive performance. Sports Medicine (Auckland, N.Z.), 32(9), 555-566.

Brown, K. W., \& Ryan, R. M. (2003). The benefits of being present: mindfulness and its role in psychological well-being. Journal of Personality and Social Psychology, 84(4), 822-848. 
Cahn, B. R., \& Polich, J. (2013). Meditation states and traits: EEG, ERP, and neuroimaging studies. Psychology of Consciousness: Theory, Research, and Practice, 1(S), 48-96. doi:10.1037/2326-5523.1.S.48

Campbell, D. T., Stanley, J. C., \& Gage, N. L. (1963). Experimental and quasiexperimental designs for research. Boston: Houghton Mifflin.

Chan, D., \& Woollacott, M. (2007). Effects of level of meditation experience on attentional focus: Is the efficiency of executive or orientation networks improved? The Journal of Alternative and Complementary Medicine, 13(6), 651-657. doi:10.1089/acm.2007.7022

Chang, Y.-K., Chi, L., Etnier, J. L., Wang, C.-C., Chu, C.-H., \& Zhou, C. (2014). Effect of acute aerobic exercise on cognitive performance: Role of cardiovascular fitness. Psychology of Sport and Exercise, 15(5), 464-470. doi:10.1016/j.psychsport.2014.04.007

Chang, Y.-K., \& Etnier, J. L. (2014). Physical activity and cognitive functioning. In A. G. Papaioannou, D. Hackfort, A. G. (Ed) Papaioannou, \& D. (Ed) Hackfort (Eds.), Routledge companion to sport and exercise psychology: Global perspectives and fundamental concepts. (pp. 705-719). New York, NY, US: Routledge/Taylor \& Francis Group.

Chang, Y.-K., Labban, J. D., Gapin, J. I., \& Etnier, J. L. (2012). The effects of acute exercise on cognitive performance: A meta-analysis. Brain Research, 1453, 87101. doi:10.1016/j.brainres.2012.02.068

Chiesa, A., \& Serretti, A. (2009). Mindfulness-based stress reduction for stress management in healthy people: a review and meta-analysis. Journal of Alternative and Complementary Medicine (New York, N.Y.), 15(5), 593-600. doi:10.1089/acm.2008.0495

Chittaro, L., \& Vianello, A. (2014). Computer-supported mindfulness: Evaluation of a mobile thought distancing application on naive meditators. International Journal of Human-Computer Studies, 72(3), 337-348. doi:10.1016/j.ijhcs.2013.11.001

Chow, T. (2014). Mindfulness Meditation versus EEG-Alpha Neurofeedback: The Role of EEG-Alpha Enhancement in Attentional Control (Master's thesis). Retrieved from the University of Ontario Electronic Thesis and Dissertation Repository.

Colcombe, S., \& Kramer, A. F. (2003). Fitness effects on the cognitive function of older adults: A meta-analytic study. Psychological Science, 14(2), 125-130. doi:10.1111/1467-9280.t01-1-01430 
Cook, T.D. and Campbell, D.T., 1979. Quasi-Experimentation: Design \& Analysis Issues for Field Settings. Boston: Houghton Mifflin Company.

Houghton Mifflin Company. Davey, C.P. (1973). Physical exertion and mental performance. Ergonomics, 16, 595-599.

Davidson, R. J., \& Goleman, D. J. (1977). The role of attention in meditation and hypnosis: a psychobiological perspective on transformations of consciousness. The International Journal of Clinical and Experimental Hypnosis, 25(4), 291308. doi:10.1080/00207147708415986

Davranche, K., Burle, B., Audiffren, M., \& Hasbroucq, T. (2005). Information processing during physical exercise: a chronometric and electromyographic study.

Experimental Brain Research, 165(4), 532-540. doi:10.1007/s00221-005-2331-9

Debray, P., Biswas, S., Biswas, P., Saha, T., \& Pal, M. S. (2015). Effect of step up exercise on cognitive attention with Stroop test in Bengali male college students. Asian Journal of Medical Sciences, 6(6), 66-69. http://doi.org/10.3126/ajms.v6i6.12602

Deepeshwar, S., Vinchurkar, S. A., Visweswaraiah, N. K., \& Nagendra, H. R. (2015). Hemodynamic responses on prefrontal cortex related to meditation and attentional task. Frontiers in Systems Neuroscience, 8.

http://doi.org/10.3389/fnsys.2014.00252

Dontje, M. L., de Groot, M., Lengton, R. R., van der Schans, C. P., \& Krijnen, W. P. (2015). Measuring steps with the Fitbit activity tracker: an inter-device reliability study. Journal of Medical Engineering \& Technology, 39(5), 286-290. http://doi.org/10.3109/03091902.2015.1050125

Eberth, J., \& Sedlmeier, P. (2012). The Effects of Mindfulness Meditation: A MetaAnalysis. Mindfulness, 3(3), 174-189. http://doi.org/10.1007/s12671-012-0101-x

Egner, T., \& Gruzelier, J. H. (2001). Learned self-regulation of EEG frequency components affects attention and event-related brain potentials in humans. Neuroreport, 12(18), 4155-4159.

Etnier, J. L., \& Chang, Y.-K. (2009). The effect of physical activity on executive function: a brief commentary on definitions, measurement issues, and the current state of the literature. Journal of Sport \& Exercise Psychology, 31(4), 469-483.

Etnier, J. L., Salazar, W, Landers, D.M., Petuzzello, S.J., Han, M.W., \& Nowell, P. (1997). The influence of physical fitness and exercise upon cognitive functioning: A meta-analysis. Journal of Sport \& Exercise Psychology, 19(3), 249-277. 
Faul, F., Erdfelder, E., Lang, A.-G., \& Buchner, A. (2007). G*Power 3: A flexible statistical power analysis program for the social, behavioral, and biomedical sciences. Behavior Research Methods, 39, 175-191.

Ferris, L. T., Williams, J. S., \& Shen, C.-L. (2007). The effect of acute exercise on serum brain-derived neurotrophic factor levels and cognitive function. Medicine and Science in Sports and Exercise, 39(4), 728-734. http://doi.org/10.1249/mss.0b013e31802f04c7

Fery, Y.-A., \& Ferry, A. (1997). Effect of physical exhaustion on cognitive functioning. Perceptual \& Motor Skills, 84(1), 291.

Foc.us tDCS headset (2015, 27 September). Retrieved from www.foc.us.

Fox, K. C. R., Nijeboer, S., Dixon, M. L., Floman, J. L., Ellamil, M., Rumak, S. P., ... Christoff, K. (2014). Is meditation associated with altered brain structure? A systematic review and meta-analysis of morphometric neuroimaging in meditation practitioners. Neuroscience \& Biobehavioral Reviews, 43, 48-73. doi:10.1016/j.neubiorev.2014.03.016

Grossman, P., Niemann, L., Schmidt, S., \& Walach, H. (2004). Mindfulness-based stress reduction and health benefits. A meta-analysis. Journal of Psychosomatic Research, 57(1), 35-43. doi:10.1016/S0022-3999(03)00573-7

Gruzelier, J. H. (2014a). EEG-neurofeedback for optimising performance. I: A review of cognitive and affective outcome in healthy participants. Neuroscience \& Biobehavioral Reviews, 44, 124-141. doi:10.1016/j.neubiorev.2013.09.015

Gruzelier, J. H. (2014b). EEG-neurofeedback for optimising performance. III: a review of methodological and theoretical considerations. Neuroscience and Biobehavioral Reviews, 44, 159-182. doi:10.1016/j.neubiorev.2014.03.015

Gutin, B. (1973). Exercise-Induced Activation and Human Performance: A Review. Research Quarterly. American Association for Health, Physical Education and Recreation, 44(3), 256-268. doi:10.1080/10671188.1973.10615204

Hillman, C. H., Erickson, K. I., \& Kramer, A. F. (2008). Be smart, exercise your heart: exercise effects on brain and cognition. Nature Reviews Neuroscience, 9(1), 5865. doi:10.1038/nrn2298

Huttermann, S., \& Memmert, D. (2014). Does the inverted-u function disappear in expert athletes? An analysis of the attentional behavior under physical exercise of athletes and non-athletes. Physiology \& Behavior, 131, 87-92.

doi:10.1016/j.physbeh.2014.04.020 
Isaacs, K. R., Anderson, B. J., Alcantara, A. A., Black, J. E., \& Greenough, W. T. (1992). Exercise and the brain: angiogenesis in the adult rat cerebellum after vigorous physical activity and motor skill learning. Journal of Cerebral Blood Flow and Metabolism, 12(1), 110-119. doi:10.1038/jcbfm.1992.14

Kabat-Zinn, J. (1982). An outpatient program in behavioral medicine for chronic pain patients based on the practice of mindfulness meditation: Theoretical considerations and preliminary results. General Hospital Psychiatry, 4(1), 33-47.

Kabat-Zinn, J. (1990). Full Catastrophe Living: Using the Wisdom of Your Body and Mind to Face Stress, Pain, and Illness. New York, N.Y: Delta.

Kahneman, D. (1973). Attention and Effort. Englewood Cliffs, N.J: Prentice Hall.

Kamijo, K., Nishihira, Y., Higashiura, T., \& Kuroiwa, K. (2007). The interactive effect of exercise intensity and task difficulty on human cognitive processing. International Journal of Psychophysiology, 65(2), 114-121. doi:10.1016/j.ijpsycho.2007.04.001

Kamijo, K., Nishihira, Y., Hatta, A., Kaneda, T., Kida, T., Higashiura, T., \& Kuroiwa, K. (2004). Changes in arousal level by differential exercise intensity. Clinical Neurophysiology, 115(12), 2693-2698. doi:10.1016/j.clinph.2004.06.016

Kamiya, J. (1968). Conscious control of brain waves. Psychology Today, 1, 57-60.

Kamiya, J. (1969). Operant control of the EEG alpha rhythm and some of its reported effects on consciousness. In C.T. Tart (Ed.) Altered States of Consciousness (pp. 519-529). New York: Wiley.

Klimesch, W. (1999). EEG alpha and theta oscillations reflect cognitive and memory performance: a review and analysis. Brain Research Reviews, 29(2-3), 169-195. doi:10.1016/S0165-0173(98)00056-3

Kluetsch, R. C., Ros, T., Théberge, J., Frewen, P. A., Calhoun, V. D., Schmahl, C., Jetly, R., Lanius, R. A. (2014). Plastic modulation of PTSD resting-state networks and subjective wellbeing by EEG neurofeedback. Acta Psychiatrica Scandinavica, 130(2), 123-136. doi:10.1111/acps.12229

Kristeller, J. L., \& Rikhye, K. (2008). Meditative traditions and contemporary psychology. In K. R. Rao, A. C. Paranjpe, \& A. K. Dalal (Eds.), Handbook of Indian psychology. (pp. 506-538). New Delhi, India: Cambridge University Press India/Foundation Books. 
Kubesch, S., Walk, L., Spitzer, M., Kammer, T., Lainburg, A., Heim, R., \& Hille, K. (2009). A 30-minute physical education program improves students' executive attention. Mind, Brain, and Education, 3(4), 235-242. doi:10.1111/j.1751228X.2009.01076.X

Kudesia, R. S., \& Nyima, V. T. (2014). Mindfulness Contextualized: An Integration of Buddhist and Neuropsychological Approaches to Cognition. Mindfulness, 1-16. doi:10.1007/s 12671-014-0337-8

Labelle, V., Bosquet, L., Mekary, S., \& Bherer, L. (2013). Decline in executive control during acute bouts of exercise as a function of exercise intensity and fitness level. Brain \& Cognition, 81(1), 10-17. doi:10.1016/j.bandc.2012.10.001

Lai, C., MacNeil, B., \& Frewen, P. (2014). A comparison of the attentional effects of single-session mindfulness meditation and fp-heg neurofeedback in novices. Mindfulness. doi:10.1007/s12671-014-0347-6

Lambourne, K., \& Tomporowski, P. (2010). The effect of exercise-induced arousal on cognitive task performance: a meta-regression analysis. Brain Research, 1341, 12-24. doi:10.1016/j.brainres.2010.03.091

Lee, J. K., \& Orsillo, S. M. (2014). Investigating cognitive flexibility as a potential mechanism of mindfulness in Generalized Anxiety Disorder. Journal of Behavior Therapy and Experimental Psychiatry, 45(1), 208-216. http://doi.org/10.1016/j.jbtep.2013.10.008

Lubar, J. O., \& Lubar, J. F. (1984). Electroencephalographic biofeedback of SMR and beta for treatment of attention deficit disorders in a clinical setting. Biofeedback and Self-Regulation, 9(1), 1-23.

Lubar, J. F., \& Shouse, M. N. (1976). EEG and behavioral changes in a hyperkinetic child concurrent with training of the sensorimotor rhythm (SMR): A preliminary report. Biofeedback and Self-Regulation, 1(3), 293-306.

Ma, J. K., Le Mare, L., \& Gurd, B. J. (2015). Four minutes of in-class high-intensity interval activity improves selective attention in 9- to 11-year olds. Applied Physiology, Nutrition \& Metabolism, 40(3), 238-244. http://doi.org/10.1139/apnm-2014-0309

Markowska, A. (2013) Attention processes in mindfulness: The influence of mindfulness intervention on performing Stroop based tasks. Acta Neuropsychologica, 11(4), 333-344. 
McMorris, T., \& Hale, B. J. (2012). Differential effects of differing intensities of acute exercise on speed and accuracy of cognition: a meta-analytical investigation. Brain and Cognition, 80(3), 338-351. doi:10.1016/j.bandc.2012.09.001

Melby-Lervag, M., \& Hulme, C. (2013). Is working memory training effective? A metaanalytic review. Developmental Psychology, 49(2), 270-291. http://doi.org/10.1037/a0028228

MindWave by NeuroSky, Inc. (2015, 27 September). Retrieved from http://store.neurosky.com/products/mindwave-1.

Monleón, C., Ballester, R., Sanchis, C., Llorens, F., Martín, M., \& Pablos, A. (2015). The effects of eight-month physical activity intervention on vigilance performance in adult obese population. Journal of Motor Behavior, 47(6), 476-482. http://doi.org/10.1080/00222895.2015.1012580

Moore, A., \& Malinowski, P. (2009). Meditation, mindfulness and cognitive flexibility. Consciousness and Cognition, 18(1), 176-186. doi:10.1016/j.concog.2008.12.008

Muse by InteraXon Inc. (2015, 2 July). Retrieved from www.choosemuse.com.

Muse Calm. (2014, 8 November) Retrieved from https:/itunes.apple.com/us/app/musecalm/id849841170?mt=8.

Neeper, S. A., Gómez-Pinilla, F., Choi, J., \& Cotman, C. (1995). Exercise and brain neurotrophins. Nature, 373(6510), 109. doi:10.1038/373109a0

Nofuji, Y., Suwa, M., Sasaki, H., Ichimiya, A., Nishichi, R., \& Kumagai, S. (2012). Different circulating brain-derived neurotrophic factor responses to acute exercise between physically active and sedentary subjects. Journal of Sports Science \& Medicine, 11(1), 83-88.

Pérez, L., Padilla, C., Parmentier, F. B. R., \& Andrés, P. (2014). The Effects of Chronic Exercise on Attentional Networks. PLoS ONE, 9(7), 1-8. doi:10.1371/journal.pone.0101478

Rabipour, S., \& Raz, A. (2012). Training the brain: Fact and fad in cognitive and behavioral remediation. Brain and Cognition, 79(2), 159-179.

doi:10.1016/j.bandc.2012.02.006

Ros, T., Théberge, J., Frewen, P. A., Kluetsch, R., Densmore, M., Calhoun, V. D., \& Lanius, R. A. (2013). Mind over chatter: Plastic up-regulation of the fMRI salience network directly after EEG neurofeedback. NeuroImage, 65, 324-335. doi:10.1016/j.neuroimage.2012.09.046 
Sedlmeier, P., Eberth, J., Schwarz, M., Zimmermann, D., Haarig, F., Jaeger, S., \& Kunze, S. (2012). The psychological effects of meditation: a meta-analysis. Psychological Bulletin, 138(6), 1139-1171. doi:10.1037/a0028168

Seligman, M. E. P. (2006). Learned Optimism: How to Change Your Mind and Your Life. New York: Vintage.

Sibley, B. A., \& Etnier, J. L. (2003). The Relationship Between Physical Activity and Cognition in Children: A Meta-Analysis. Pediatric Exercise Science, 15(3), 243.

Sibley, B. A., Etnier, J. L., \& Le Masurier, G. C. (2006). Effects of an Acute Bout of Exercise on Cognitive Aspects of Stroop Performance. Journal of Sport \& Exercise Psychology, 28(3), 285-299.

Slagter, H. A., Davidson, R. J., \& Lutz, A. (2011). Mental training as a tool in the neuroscientific study of brain and cognitive plasticity. Frontiers in Human Neuroscience, 5, 17. doi:10.3389/fnhum.2011.00017

Sparrow, W. A., \& Wright, B. J. (1993). Effect of physical exercise on the performance of cognitive tasks. Perceptual and Motor Skills, 77(2), 675-679. doi:10.2466/pms.1993.77.2.675

Stein, E., Crowley, S., Dunnam, M., \& Anderson-Hanley, C. (2014). B64Neuropsychological Benefits of Interactive Mental and Physical Exercise. Archives of Clinical Neuropsychology: The Official Journal of the National Academy of Neuropsychologists, 29(6), 561. http://doi.org/10.1093/arclin/acu038.152

Sterman, M. B., Howe, R. C., \& MacDonald, L. R. (1970). Facilitation of spindle-burst sleep by conditioning of electroencephalographic activity while awake. Science (New York, N.Y.), 167(3921), 1146-1148.

Sterman, M. B., Macdonald, L. R., \& Stone, R. K. (1974). Biofeedback Training of the Sensorimotor Electroencephalogram Rhythm in Man: Effects on Epilepsy. Epilepsia, 15(3), 395-416. doi:10.1111/j.1528-1157.1974.tb04016.x

Sterman, M. B., \& Wyrwicka, W. (1967). EEG correlates of sleep: Evidence for separate forebrain substrates. Brain Research, 6(1), 143-163.

Stroop, R.J. (1935). Studies of interference in serial verbal reactions. Journal of Experimental Psychology, 18(6), 643-662. doi:10.1037/h0054651

Tanaka, H., Monahan, K. D., \& Seals, D. R. (2001). Age-predicted maximal heart rate revisited. Journal of the American College of Cardiology, 37(1), 153-156. 
Tomasino, B., Fregona, S., Skrap, M., \& Fabbro, F. (2013). Meditation-related activations are modulated by the practices needed to obtain it and by the expertise: an ALE meta-analysis study. Frontiers in Human Neuroscience, 6, 346. http://doi.org/10.3389/fnhum.2012.00346

Tomporowski, P. D. (2003). Effects of acute bouts of exercise on cognition. Acta Psychologica, 112(3), 297-324.

van Praag, H., Kempermann, G., \& Gage, F. H. (1999). Running increases cell proliferation and neurogenesis in the adult mouse dentate gyrus. Nature Neuroscience, 2(3), 266-270. http://doi.org/10.1038/6368

Vernon, D. J. (2005). Can neurofeedback training enhance performance? An evaluation of the evidence with implications for future research. Applied Psychophysiology and Biofeedback, 30(4), 347-364. doi:10.1007/s10484-005-8421-4

Vidyarthi, J., \& Riecke, B. E. (2014). Interactively mediating experiences of mindfulness meditation. International Journal of Human-Computer Studies, 72(8-9), 674688. doi:10.1016/j.ijhcs.2014.01.006

Wallace, B. A. (1999). The Buddhist tradition of Samatha: Methods for refining and examining consciousness. Journal of Consciousness Studies, 6(2-3), 175-187.

Wallace, B. A., \& Shapiro, S. L. (2006). Mental balance and well-being: Building bridges between Buddhism and Western psychology. American Psychologist, 61(7), 690701. doi:10.1037/0003-066X.61.7.690

Walsh, R. (1982). The Original Goals of Meditation. American Journal of Psychiatry, 139(11), 1525-b.

Wang, J.-R., \& Hsieh, S. (2013). Neurofeedback training improves attention and working memory performance. Clinical Neurophysiology: Official Journal of the International Federation of Clinical Neurophysiology, 124(12), 2406-2420. doi:10.1016/j.clinph.2013.05.020

Wenk-Sormaz, H. (2005). Meditation can reduce habitual responding. Advances in MindBody Medicine, 21(3-4), 33-49.

Yerkes, R. M., \& Dodson, J. D. (1908). The relation of strength of stimulus to rapidity of habit-formation. Journal of Comparative Neurology and Psychology, 18(5), 459482. doi:10.1002/cne.920180503 
Zoladz, J. A., Pilc, A., Majerczak, J., Grandys, M., Zapart-Bukowska, J., \& Duda, K. (2008). Endurance training increases plasma brain-derived neurotrophic factor concentration in young healthy men. Journal of Physiology and Pharmacology, 59 Suppl 7, 119-132. 


\section{Appendix A}

\section{Physical Activity Readiness Questionnaire (PAR-Q)} Questionnaire - PAR-Q (revised 2002)
Physical Activity Readiness

\section{PAR-Q \& YOU}

(A Questionnaire for People Aged 15 to 69)

Regular physical activity is fun and healthy, and increasingly more people are starting to become more active every day. Being more active is very safe for most people. However, some people should check with their doctor before they start becoming much more physically active.

If you are planning to become much more physically active than you are now, start by answering the seven questions in the box below. If you are between the ages of 15 and 69, the PAR-Q will tell you if you should check with your doctor before you start. If you are over 69 years of age, and you are not used to being very active, check with your doctor.

Common sense is your best guide when you answer these questions. Please read the questions carefully and answer each one honestly: check YES or NO

\begin{tabular}{|c|c|c|c|}
\hline YES & No & & \\
\hline$\square$ & $\square$ & 1. & $\begin{array}{l}\text { Has your doctor ever said that you have a heart condition and that you should only do physical activity } \\
\text { recommended by a doctor? }\end{array}$ \\
\hline$\square$ & $\square$ & 2. & Do you feel pain in your chest when you do physical activity? \\
\hline$\square$ & $\square$ & 3. & In the past month, have you had chest pain when you were not doing physical activity? \\
\hline$\square$ & $\square$ & 4. & Do you lose your balance because of dizziness or do you ever lose consciousness? \\
\hline$\square$ & $\square$ & 5. & $\begin{array}{l}\text { Do you have a bone or joint problem (for example, back, knee or hip) that could be made worse by a } \\
\text { change in your physical activity? }\end{array}$ \\
\hline$\square$ & $\square$ & 6. & $\begin{array}{l}\text { Is your doctor currently prescribing drugs (for example, water pills) for your blood pressure or heart con- } \\
\text { dition? }\end{array}$ \\
\hline$\square$ & $\square$ & 7. & Do you know of any other reason why you should not do physical activity? \\
\hline
\end{tabular}

If

\section{YES to one or more questions}

you

Talk with your doctor by phone or in person BEFORE you start becoming much more physically active or BEFORE you have a fitness appraisal. Tell your doctor about the PAR-Q and which questions you answered YES.

- You may be able to do any activity you want — as long as you start slowly and build up gradually. Or, you may need to restrict your activities to

answered

\section{NO to all questions}

If you answered NO honestly to all PAR-Q questions, you can be reasonably sure that you can: - start becoming much more physically active - begin slowly and build up gradually. This is the safest and easiest way to go.

- take part in a fitness appraisal - this is an excellent way to determine your basic fitness so that you can plan the best way for you to live actively. It is also highly recommended that you have your blood pressure evaluated. If your reading is over 144/94, talk with your doctor before you start becoming much more physically active.

Informed Use of the PAR-Q: The Canadian Society for Exercise Physiology, Health Canada, and their agents assume no liability for persons who undertake physical activity, and if in doubt after completing this questionnaire, consult your doctor prior to physical activity.

\begin{tabular}{|l}
\hline No changes permitted. You are encouraged to photocopy the PAR-Q but only if you use the entire form. \\
\hline NOTE: If the PAR-Q is being given to a person before he or she participates in a physical activity program or a fitness appraisal, this section may be used for legal or administrative purposes.
\end{tabular}

"I have read, understood and completed this questionnaire. Any questions I had were answered to my full satisfaction."

NAME

SIGNATURE

DATE

SIGNATURE OF PARENT

or GUARDIAN (for participants under the age of majority)

WITNESS

Note: This physical activity clearance is valid for a maximum of 12 months from the date it is completed and

becomes invalid if your condition changes so that you would answer YES to any of the seven questions.

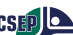

(c) Canadian Society for Exercise Physiology Supported by: Health Santé

continued on other side... 


\section{Appendix B}

Pre-Experiment Questionnaire

Please answer the following questions to the best of your ability.

1. What is your age range?

○ 17 or younger

- $18-25$

○ 26-35

○ $36-45$

○ $46-55$

○ $55-69$

○ 70 or older

2. What is your gender?

o Female

○ Male

- Prefer not to answer

3. How frequently do you exercise?

- I don't exercise on a regular basis

- 1 day per week

- 2 days per week

○ 3 days per week

- 4 days per week

- 5 days per week

- 6 days per week

- 7 days per week

4. How long do you exercise in a single session?

- I don't exercise on a regular basis

- Less than 20 minutes

- 20-30 minutes

- 30-45 minutes

- More than 60 minutes

5. What kind of exercise do you engage in on a weekly basis? (Check all that apply)

- Moderate intensity aerobic exercise, such as brisk walking or cycling on level ground

口 High intensity exercise, such as running or swimming laps

口 Strength training, such as lifting weights or yoga

口 Stretching 
6. Do you currently practice any type of meditation?

○ Yes

○ No

7. Are you colorblind?
- Yes
○ No 


\title{
Appendix C
}

\author{
Participant consent form
}

REQUEST FOR YOUR PARTICPATION IN RESEARCH

\section{PHYSICAL EXERCISE, MEDITATION, NEUROFEEDBACK, AND COGNITIVE PERFORMANCE}

\section{NAME OF THE RESEARCHER}

Claire Balgemann, San Jose State University graduate student

Please take your time in deciding if you would like to participate. You must be between the ages of 18 and 69 years to participate in this study.

\section{PURPOSE}

The purpose of this research is to investigate how participation in a single bout of moderateintensity exercise, mindfulness meditation, or mindfulness meditation neurofeedback affects cognitive performance. The results of this study will contribute to the body of knowledge about cognitive performance and may be used to improve cognitive performance-enhancing devices.

\section{PROCEDURES}

Sessions will last approximately 40-50 minutes and will take place at a climate-controlled room in San Jose State University. During that time, you will spend 20 minutes either exercising on a stationary bicycle, engaging in mindfulness meditation, or engaging in mindfulness meditation neurofeedback with a Muse brain-sensing headband. Depending on which group you are assigned to, you will be provided detailed instructions for how to monitor your exercise so that it is within moderate-intensity range or instructions for how to use the Muse brain-sensing headband. The Muse brain-sensing headband is a lightweight, non-invasive device that will be placed on your head to read your brainwaves. It cannot read your thoughts or alter your brainwaves and will not allow you to control objects with your thoughts. Muse simply reads your brainwaves similar to how a heart rate monitor reads your heartbeat.

You will also be asked to complete a cognitive test that will require you to read from a computer monitor and say words aloud. If you wear contact lenses or glasses to read from a computer screen, please wear them for this part of the experiment.

\section{POTENTIAL RISKS}

There are very few risks from participating in this study. Potential risks include the possibility of physical injury while exercising for 20 minutes at moderate-intensity on a stationary bicycle. Using the Muse brain-sensing headband presents no more than minimal risks. The headband is like a heart rate monitor; it is non-invasive and monitors brainwaves from your scalp similar to how a heart rate monitor reads your heart beat. Muse is not capable of altering a your thoughts or 
brainwave patterns. The probability and magnitude of harm or discomfort is no greater than what would be encountered in daily life.

\section{POTENTIAL BENEFITS}

By completing this study, you may experience short-term wellbeing associated with exercising, participating in mindfulness meditation, or mindfulness meditation neurofeedback. You may also help to contribute to the body of knowledge regarding how exercise, mindfulness meditation, and mindfulness meditation neurofeedback affect cognitive performance, which may improve future cognitive performance-enhancing devices.

\section{COMPENSATION}

Students in the psychology research subject pool will receive partial credit towards Psychology 001. All participants may enter a raffle to win one of three \$25 Amazon gift cards. No other compensation is provided.

\section{CONFIDENTIALITY}

The results of this study will not be associated with you in any way. The investigator is required to keep a copy of this informed consent document as well as the documents you previously filled out (the Physical Activity Readiness Questionnaire and the Pre-Experiment Questionnaire), but they will be kept separate from the study results. No records are kept that allow your name to be associated with your data or responses in the study. Your responses will be kept anonymous.

\section{PARTICIPANT RIGHTS}

Your participation in this study is completely voluntary. You can refuse to participate in the entire study or any part of the study without any negative effect on your relations with San Jose State University. You also have the right to skip any question you do not wish to answer. This consent form is not a contract. It is a written explanation of what will happen during the study if you decide to participate. You will not waive any rights if you choose not to participate, and there is no penalty for stopping your participation in the study.

\section{QUESTIONS OR PROBLEMS}

You are encouraged to ask questions at any time during this study.

- For further information about the study, please contact Claire Balgemann: claire.balgemann@gmail.com.

- Complaints about the research may be presented to Dr. Emily Wughalter, Professor and Thesis Chair, Graduate Program in Human Factors \& Ergonomics and Department of Kinesiology: emily.wughalter@sjsu.edu.

- For questions about participants' rights or if you feel you have been harmed in any way by your participation in this study, please contact Dr. Pamela Stacks, Associate Vice President of Graduate Studies and Research, San Jose State University, at 408-924-2427. 


\section{SIGNATURES}

Your signature indicates that you voluntarily agree to be a part of the study, that the details of the study have been explained to you, that you have been given time to read this document, and that your questions have been answered. You will receive a copy of this consent form for your records.

\section{Participant Signature}

Participant's Name (printed)

Participant's Signature

Date

\section{Researcher Statement}

I certify that the participant has been given adequate time to learn about the study and ask questions. It is my opinion that the participant understands his/her rights and the purpose, risks, benefits, and procedures of the research and has voluntarily agreed to participate. 


\section{Appendix D}

\section{IRB Approval Letter}

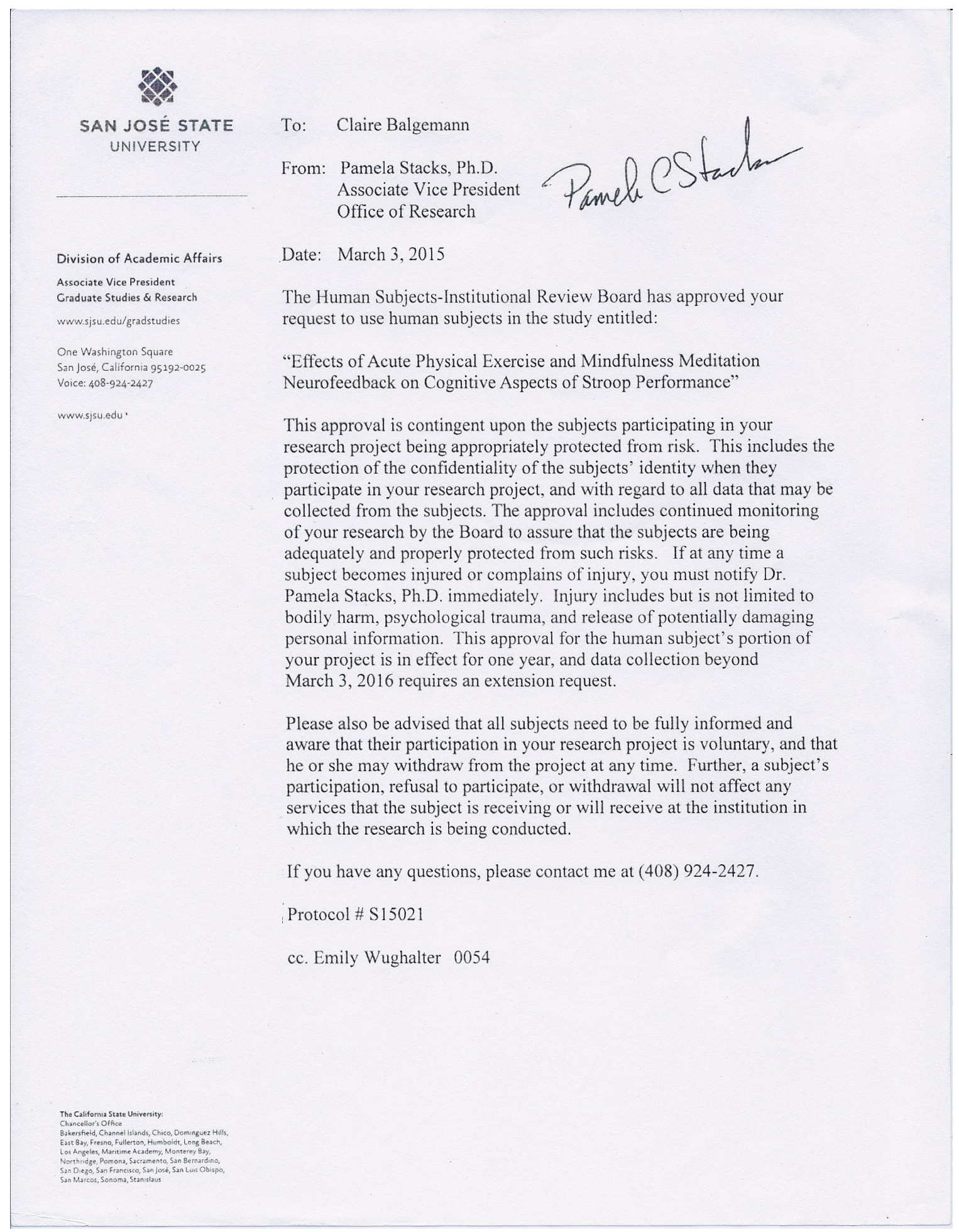

